\title{
Comprehensive single-cell gene and isoform expression analysis reveals signatures of ageing in haematopoietic stem and progenitor cells
}

\section{Laura Mincarelli}

Earlham Institute https://orcid.org/0000-0002-9222-2676

Vladimir Uzun

Earlham Institute

David Wright

Earlham Institute

\section{Stuart Rushworth}

University of East Anglia https://orcid.org/0000-0002-3711-7558

\section{Wilfried Haerty}

Earlham Institute

lain Macaulay ( $\sim$ iain.macaulay@earlham.ac.uk)

Earlham Institute

\section{Article}

Keywords: isoform expression, gene expression, haematopoietic stem cells, progenitor cells

Posted Date: February 26th, 2021

DOl: https://doi.org/10.21203/rs.3.rs-151546/v1

License: (1) This work is licensed under a Creative Commons Attribution 4.0 International License. Read Full License 
Comprehensive single-cell gene and isoform expression analysis reveals signatures of ageing in haematopoietic stem and progenitor cells

Laura Mincarelli ${ }^{1}$, Vladimir Uzun ${ }^{1}$, David Wright ${ }^{1}$, Stuart A. Rushworth², Wilfried Haerty ${ }^{1 *}$, lain

C. Macaulay ${ }^{1^{*}}$

Affiliations

${ }^{1}$ Earlham Institute, Norwich Research Park, Norwich, NR4 7UZ

${ }^{2}$ Norwich Medical School, The University of East Anglia, Norwich Research Park, Norwich, United Kingdom 


\begin{abstract}
Single-cell approaches have revealed that the haematopoietic hierarchy is a continuum of differentiation, from stem cell to committed progenitor, marked by changes in gene expression. However, many of these approaches neglect isoform level information, and thus do not capture the extent and effect of alternative splicing within the system. Here, we present the first integrated short- and long-read single-cell RNA-seq of haematopoietic stem and progenitor cells. We demonstrate that over half of genes detected in standard short-read single-cell analyses are expressed as multiple, often functionally distinct, isoforms. This includes many transcription factors and key cytokine receptors, and in particular the Thrombopoietin receptor $\mathrm{Mpl}$, which displays complex isoform expression patterns between individual hematopoietic stem cells. The dataset further reveals novel signatures of hematopoietic ageing, including a global increase in IncRNA expression. Strikingly, the longread sequencing enables us to observe aberrant expression of full-length VJ-rearranged immunoglobulin kappa transcripts in aged haematopoietic stem cells, prior to lymphoid commitment. Integrating single cell and cell-type specific isoform landscape in normal and aged hematopoiesis provides a new reference for accurate molecular profiling of heterogeneous tissues, as well as novel insights into transcriptional complexity, cell-type specific splicing events and effects of ageing.
\end{abstract}




\section{Introduction}

Single-cell RNA-seq (scRNA-seq) technologies are now applied to a broad spectrum of biological systems ${ }^{1}$ with particular impact in the study of stem cell and developmental biology ${ }^{2-4}$. With the advance of short-read technologies capable of analysing many thousands of cells in a single experiment, it has become possible to identify cell types and state transitions in complex biological systems. In particular, scRNA-seq has highlighted the continuous nature of haematopoietic hierarchy. Cell types, previously thought of as discrete entities, have been shown to exist in a continuum of states from stem cell to mature progenitor ${ }^{5}$. Investigating the regulatory events that occur in these state transitions, and how they change with age, is central to the understanding of the regenerative potential of stem cells in health and disease.

Alternative splicing (AS) of mRNA transcripts is a mechanism by which several isoforms can be generated from individual genomic locus, enabling significant increases in transcriptomic and proteomic complexity. AS can affect many aspects of gene expression, including transcript export from the nucleus, transcript stability, and critically the production of functionally distinct protein isoforms. AS is thought to occur in at least $62 \%$ of multi-exonic genes in mouse ${ }^{6}$ and up to $95 \%$ of multi-exonic genes in human ${ }^{7}$. Increasingly, there is an understanding that isoform (co-)expression in tissues and cells can reveal previously unseen complexities in cell signalling responses, as was demonstrated recently for G-protein coupled receptors ${ }^{8}$.

In haematopoiesis, substantial levels of AS have been observed in sorted populations of stem and progenitor cells ${ }^{9,10}$, but in general AS remains overlooked in scRNA-seq studies. Because the vast majority of scRNA-seq approaches target just 3' or 5' transcript ends, AS events are unlikely to be captured and thus an entire class of biologically important information about celland cell-type specific gene expression is lost. Advances in long-read sequencing technologies have enabled unequivocal detection of AS isoforms ${ }^{11}$. Here, we have developed and applied integrated parallel short- (Illumina) and long-read (Pacific Biosciences; PacBio) sequencing of single-cell libraries generated using the 10X Genomics Chromium. This enables parallel profiling cellular diversity, gene expression and AS events in the mouse haematopoietic system.

To demonstrate the utility of this approach, we performed parallel gene and isoform expression analysis on haematopoietic stem and progenitor cells isolated from young and aged C57/BL6 mice. We generated cell-barcoded cDNA using the 10X Genomics platform, and Illumina 
sequencing of this cDNA to reveal cell states, gene expression, and cell frequency changes associated with ageing. Parallel PacBio sequencing of this full-length cDNA and integration of the cell barcodes enabled assignment of AS isoforms to individual cells and cell-type clusters generated from the short-read data (Fig 1A, Supplementary Fig 1). We demonstrate that AS is readily detectable by long-read sequencing of scRNA-seq libraries and that this integrated approach can identify novel aspects of gene expression in cell differentiation and ageing. We observe that functionally divergent isoforms, undetectable by short-read sequencing alone, are common throughout haematopoiesis, and thus that isoform-level analysis is critical for the understanding of cell states and their response to signals. 


\section{Results}

\section{Generation of short- and long- read single cell RNA seq libraries}

Using Fluorescence Activated Cell Sorting (FACS) we isolated the Lineage negative, cKit (Cd117) positive (LK) cell fraction of mouse bone marrow cells, a population containing stem and progenitor cells ${ }^{12}$ from young ( 8 week) and aged (72 week) mice. We generated standard single-cell sequencing libraries from these populations, which revealed the diversity of cell types present within the 8,000 LK cells passing quality control (Fig 1B).

Analysis of this data identified 15 subclusters within the LK population, including long-term haematopoietic stem cells (HSCs), here associated with Procr (Cd201) expression ${ }^{13}$, as well as intermediate and committed progenitor cells identifiable by distinct marker gene expression (Fig 1B, Supplementary Table 1). This matches well the diversity expected from FACSbased phenotypic analysis of the same population ${ }^{12}$. Small numbers of mature B-cells, myeloid cells and mast cells were also observed, but were transcriptionally very distinct from the main stem and progenitor cell cluster, and most likely represent low level contamination with mature cells.

In order to further examine transcriptional heterogeneity in the haematopoietic system, we performed long-read PacBio sequencing (IsoSeq) on the cDNA pools generated from the 10X platform, similar to an approach recently applied in cerebellar cells ${ }^{14}$. This protocol, taking advantage of the cell barcoding technology used in 10X Genomics library preparation, enables isoform identification and association with cell populations, and potentially even single-cells, through integration of the long- and short- read data.

A detailed breakdown of the PacBio sequencing statistics is presented in Supplementary Table 2. In brief, PacBio sequencing yielded a total of 17.9 million circular consensus sequencing (CCS) reads with a median read length of 1471 bases (Fig 2A). These reads mapped to an average of 16,427 genes per sample, representing an average of 33,345 transcripts per sample and an average of 31 reads per transcript. Transcript coverage averaged $74 \%$ (Fig 2B), and alternative isoforms were detected for $52.3 \%$ of genes (Fig $\mathbf{2 C}$ ).

We took advantage of the long-read pacbio data to annotate and explore alternative splicing events using TALON ${ }^{15}$. We identified a total of 11,013 novel transcripts supported by at least five reads and identified in at least three of the samples (44,993 novel transcripts if at least two reads and two samples). Those annotations also enabled the identification of 910 novel 
cassette exons, 4,118 and 3,465 novel alternative 5' and 3; splicing sites respectively. We also identified 143 novel junctions between previously annotated splice sites. We investigated the impact of the novel splicing events on the coding potential of the transcripts. Among the 10,576 novel transcripts arising from protein coding genes, a total of 6,747 transcripts were identified as coding, whereas 3,830 were deemed non coding (Supplementary Table 3)

Demultiplexing of the long-reads using the cell barcodes identified in the short-read analysis enabled 5.8 million (32\%) of CCS reads to be assigned to individual cells. Using the multimodal capabilities of Seurat ${ }^{16}$, we integrated short- and long-read datasets allowing side-byside comparison of gene and isoform expression from the respective platforms, and annotation of individual cells with isoform-level transcript information. A median of 411 reads, corresponding to a median of 292 transcripts, could be assigned per cell (Fig 2D and 2E). In general, the numbers of isoforms detected are too low to allow meaningful comparisons at single-cell resolution. However, with 50-500,000 long-reads per cluster, scaling linearly with the number of cells per cluster (Fig 2F-H), it is possible to associate isoform expression with cell-type clusters.

\section{Alternative Splicing in Haematopoietic Transcription Factor Networks}

To highlight the impact of additional long-read sequencing in scRNA-seq studies, we screened the dataset to identify AS events in key transcription factors (TFs) from a regulatory network derived from single-cell studies of haematopoiesis ${ }^{17}$. Our long-read sequencing detected 28 (of 31) TFs in that network (Fig 3A), including Lmo2, Gata2, Ldb1 and Tal1, which when translated form protein complexes that regulate cell fate decisions across hematopoiesis.

Three predominant isoforms were detected for Lmo2, Lmo2-202, Lmo2-203 and Lmo2-208, each of which encodes a protein differing in length (228, 220 and 158 amino acids, respectively) with progressive truncation from the $\mathrm{N}$-terminus. Additionally, a novel, in-frame variant of $L m o 2$ with a 297 bp exon was also detected and supported by 27 long-reads (Supplementary Fig 2). In our data, while Lmo2-202 and 203 are widely expressed, the

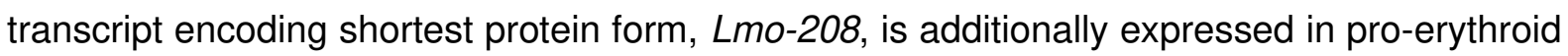
cells, megakaryocytes and mast cells (Fig 3B). In human cell lines, long- and short- protein isoforms of Lmo2 (equivalent to the proteins encoded by Lmo2-202 and -203) have been shown to have distinct functional roles in the formation of TF complexes and regulation of gene expression ${ }^{18}$. Gata2 expression, which in our short read data is restricted to stem and progenitor cells, mast cell and early megakaryocyte and erythroid progenitor populations, consists of two main isoforms, Gata2-201 and Gata2-202 (Fig 3C). They are translated into 
the same protein but are differentiated by the usage of distinct distal exons, and have previously been shown to exhibit some lineage specific expression ${ }^{19}$. Here, both isoforms are most abundant in the mast cell population, with Gata2-202 showing more restricted expression to this cell type (Fig $\mathbf{3 C}$ ). We similarly observe the expression of multiple isoforms of $L d b 1$ (Fig 3D) and Tal1 (Fig 3E).

\section{Alternative Splicing and Cytokine receptors}

Transmembrane proteins can determine the cellular response to its environment, particularly regulatory cytokines. We screened the long-read data for isoform expression of a panel of cytokine receptors (Fig 4A). Two predominant isoforms of the stem cell factor (SCF) receptor Kit (Kit-201 and Kit-204) were observed to be expressed in virtually all cell types (Fig 4B). These isoforms encode proteins differing in length by 4 amino acids (KGNN) which, when absent, results in the loss of a low-complexity region in the juxtamembrane extracellular region of the protein. The human equivalent of these variants have been shown to display distinct signalling activities ${ }^{20-22}$.

Four isoforms of $\mathrm{Mpl}$, the gene encoding the thrombopoietin (ThPO) receptor ${ }^{23}$, were detectable in both stem cells and megakaryocytes (Fig 4C). The primary isoform, Mpl-201 encodes the transmembrane $\mathrm{Mpl}$ receptor. Mpl-202 encodes a shortened version of the protein, lacking 8 amino acids in the extracellular domain. Mpl-204, which lacks exons 9 and 10 completely, encodes a truncated protein with no transmembrane domain. This isoform is detectable in the stem cell population, and functional studies have indicated that it has an inhibitory role on normal Mpl signalling ${ }^{23}$.

Using junction-targeting qPCR, we examined the distribution of Mpl isoforms in single FACS isolated HSCs (LSK Cd150+ Cd48- Cd34-) (Fig 4D). This demonstrated that individual HSCs frequently express more than one isoform, and in some cases three or four isoforms could be detected in the same single cell. Mpl-202 is the most common isoform but is frequently coexpressed with $\mathrm{Mpl}-204$ and also occasionally with $\mathrm{Mpl}$-203. The observation that individual HSCs can express more than one isoform of this key cytokine receptor suggests that ThPO signalling can be distinctively regulated in the functionally heterogeneous or lineage-primed stem cell pool. This highlights the critical need to understand not just gene, but isoform expression in single-cell studies. 


\section{Signatures of ageing}

We integrated short- and long-read data to reveal age-associated signatures in cell type abundance, gene and isoform expression. While the majority of cell type abundances were unchanged between 8- and 72-week old mice, there was an increased abundance of phenotypic LT-HSCs (Fig 5A-C) in keeping with previous findings based on stem cells defined by protein marker expression (Lineage negative, Sca-1 positive, cKit positive (LSK) CD34CD48- CD150+ cells) ${ }^{24}$.

To identify transcriptional signatures associated with ageing across the haematopoietic hierarchy, we performed differential gene expression analysis in each population using the short-read sequencing data (Fig 5D). We observed downregulation of genes encoding the cytosolic ribosomal components Rp/35 and Uba52 in stem cells and all progenitors. A decrease in expression of UBA52 and RPL35 genes has also been observed in aged human peripheral blood ${ }^{25}$. The cytosolic ribosomal subunit Rpl35 and the ubiquitin-ribosomal hybrid encoding gene Uba52 ensure protein homeostasis and efficient turnover of defective proteins. Deficiency of Uba52 results in reduction of protein synthesis and cell cycle arrest ${ }^{26}$. Interestingly, functional decline of ubiquitin-dependent protein clearance with increasing age reinforces the ageing process through accumulation of damaged and aggregated proteins, ultimately interfering with cell function ${ }^{27}$. Specifically in the HSC population, we observed ageassociated upregulation of HSC-specific genes including Sult1a1 and Nupr1 in addition to panhematopoietic upregulated genes including lgkc. We also observed downregulation of Rp/35, Uba52, Tmsb10, Gpx1, Plac8 and Cd34 (Fig 5E, Supplementary Table 4). Sult1a1 and Nupr1 are highly restricted to the LT-HSC population, and are indeed almost exclusively expressed in aged LT-HSCs (Fig 5F-G) and this increase in expression was confirmed in FACS-purified HSCs (LSK Cd34- Cd48- Cd150+) from young and aged mice by qPCR (Fig $\mathbf{5 H}$ ). While Nupr1 was not detected in the long-read data, the Sult1a1-203 isoform was the predominant isoform detected, encoding a 188 aa variant of the Sult1a1 protein.

A global comparison of the long-read data from young and aged mice showed an ageassociated increase in expression of noncoding transcripts, including lgV pseudogenes, IncRNAs and transcripts with retained introns (Fig 6A). This is consistent with observations that an increased frequency of intron retention has been identified as a signature of ageing in fruitfly, mouse and human ${ }^{28,29}$.

Following the observation that lgkc transcripts were upregulated in the aged LT-HSC population, and indeed throughout the myeloid progenitors (Fig 6B), we used the long-read 
data to determine that the lgkc arising from the LT-HSC population in aged mice consisted not just of "germline" Igkc, as previously reported ${ }^{30}$, but fully VJ-recombined transcripts (Fig 6C, Supplementary Fig 3), and distinct from the upregulation of $\lg \vee$ pseudogenes (which account for only $1 \%$ of immunoglobulin reads sequenced). 


\section{Discussion}

Accurately defining isoform expression may be key to understanding functional heterogeneity in cells with similar gene expression profiles. Aberrations in AS and its regulation are often associated with ageing and disease, and thus methods to analyse isoform expression at single cell resolution will be essential to understand the full impact this can have. Here, we demonstrate that long-read sequencing can be integrated into conventional single-cell approaches to profile cell-type specific isoform expression, and to predict functional diversity in the cell's proteome. This also enables integration of previously unannotated isoforms into reference transcriptomes to allow a more accurate annotation of cell types specific isoform expression, and a better understanding of contribution of isoform dynamics to cellular function.

We demonstrate that over half the genes detected in a standard single-cell analysis of the haematopoietic hierarchy are actually present as multiple isoforms, including transcription factors and transmembrane receptors with key roles in haematopoietic differentiation. Complexes such as Lmo2/Gata2/Ldb1/Tal1 may be readily subject to regulation through isoform expression, with distinct protein isoforms having distinct roles within the overall complex. The finding that many of the transcription factors comprising established networks are present as multiple isoforms suggests an additional layer of complexity that should be taken into account, for example when building gene regulatory networks from single-cell expression data.

Similarly, it may be that AS events underlie how phenotypically highly similar cells respond differently to extrinsic signals. We detected multiple isoforms of the transcript encoding the ThPO receptor $\mathrm{Mpl}$, a key regulator of stem cell maintenance and megakaryocyte differentiation and identified that these isoforms, with functionally distinct protein products are heterogeneously expressed in phenotypic HSCs. We demonstrate for the first time that single HSCs often express more than one isoform of $\mathrm{Mpl}$ with each isoform potentially having antagonistic responses to ligand binding. Functional heterogeneity in isoform expression/coexpression could potentially have a critical role in how individual HSCs respond to ThPO, and it would be of considerable interest to understand the role this might play in both steady-state and stress haematopoiesis.

The integrated analysis of short- and long-read data further allowed the identification of multiple signatures of hematopoietic ageing. Consistent with previous reports, we observe an expansion of a transcriptionally phenotypic HSC population with ageing, while other cell populations remain largely unchanged. Furthermore, the short-read data identified Sult1a1 and Nupr1 expression to be almost exclusively expressed in aged HSCs. The genes encoding 
Nupr1 and Sult1a1 are co-localised in a $50 \mathrm{~kb}$ region of chromosome 7, and their elevated expression has previously been shown to be associated with an age-related increase in the H3K4me3 chromatin mark in HSCs ${ }^{31}$. Sult1a1 encodes for a sulfotransferase which acts on substrates including hormones and neurotransmitters, and Nupr1 has a regulatory role in cell proliferation and apoptosis, but neither has a described functional role in haematopoiesis ${ }^{31,32}$.

Upon ageing, no specific changes in isoform expression could be observed at this resolution but the long-read data did show increased expression of IncRNAs. Highly tissue-specific changes in IncRNAs expression in aged human tissues has been reported ${ }^{33}$ and abnormally elevated expression of some IncRNAs seem to relate to telomere shortening and senescence 34. Similarly, an overall increase in intron retention was observed. Perhaps the most striking signature of ageing detected through the combined use of short- and long-read sequencing was the upregulation of recombined lgkc throughout hematopoiesis, including in phenotypic HSCs. This was highly unexpected but perhaps not without precedent. Previous work has shown that unrecombined lgkc transcripts are expressed in aged LT-HSCs, possibly as a result of epigenetic dysregulation ${ }^{30}$. Interestingly, lgkc expression was also observed to be upregulated in microarray analysis of gene expression in VWF+ platelet-biased HSCs when compared with vWF- HSCs ${ }^{35}$.

The generation of these recombined transcripts is dependent on genomic rearrangements, typically seen in committed lymphoid progenitors downstream of HSCs. This finding suggests that premature activation of the recombination-activating genes Rag-1 and Rag-2, in addition to epigenetic changes in the lgkc locus, may represent a novel hallmark of ageing stem cells. There is an increasing body of evidence that the expression of recombined Ig molecules in non-lymphoid cells is possible ${ }^{36-38}$, including human CD34+ cord blood stem and progenitor cells ${ }^{39}$ and acute myeloid leukemia ${ }^{40}$ ), although the precise mechanisms of how the required structural changes in the genome enabling this can occur remain to be determined. Within the aged HSCs, there is some heterogeneity in VJ recombination, with a few dominant clones detected, for example $l g k c / l g k j 1 / l g k v 3-2$, indicating that this recombination has occurred in multiple distinct HSC, from which some have generated recombined clonal progeny which retain the HSC phenotype.

Overall, our study not only provides an unprecedented picture of cell type-specific gene and isoform expression in a variety of haematopoietic cells but also offers novel insights into transcriptional signatures of ageing in stem and progenitor cells. Our results highlight the need to further characterise isoform diversity at single cell level and to build an isoform atlas for different cell types to reveal the full extent of transcriptional heterogeneity in development, 
ageing and disease. The observation that full-length, recombined immunoglobulin expression can occur in aged HSCs is a striking finding that requires further mechanistic investigation.

With continued improvements in the throughput of long-read sequencing platforms and development of methods targeting specific cells or transcripts, we envision that long-read sequencing will enable detailed characterisation of the total landscape of isoform diversity at the single-cell level at a scale comparable to current short-read based methods. This will be applicable to large-scale atlassing studies of cellular heterogeneity, and in haematological malignancies where mutations in splicing factors are common ${ }^{41}$. 


\section{Sources of funding}

ICM is supported by a BBSRC New Investigator Grant [BB/P022073/1] and the BBSRC National Capability in Genomics and Single Cell Analysis at Earlham Institute [BB/CCG1720/1]. WH and DW are supported by the BBSRC Core Strategic Programme Grant [BB/P016774/1], and WH by a UK Medical Research Council [MR/P026028/1] award. SR is funded by the Rosetrees Trust, The Big C and the Medical Research Council [MR/T02934X/1]. Next-generation sequencing was delivered via the BBSRC National Capability in Genomics and Single Cell Analysis [BB/CCG1720/1] at Earlham Institute.

\section{Author Contributions}

LM, SAR and ICM designed and performed experiments. LM, VU, DW, WH and ICM analysed data.

\section{Corresponding Authors}

Correspondence to Wilfried Haerty (wilfried.haerty@earlham.ac.uk) and lain Macaulay (iain.macaulay@earlham.ac.uk)

\section{Competing interests.}

No competing interest to declare 


\section{Methods}

\section{Stem and Progenitor cell isolation}

All animal work in this study was carried out in accordance with regulations set by the United Kingdom Home Office and the Animal Scientific Procedures Act of 1986. Bone marrow was isolated from spine, femora, tibiae, and ilia of 8 weeks and 72 weeks old C57BL/6 mice. Red blood cell depletion was performed with ammonium chloride lysis (STEMCELL Technologies) and lineage negative cells were isolated using the EasySep Mouse Hematopoietic Progenitor Cell Isolation Kit (STEMCELL Technologies).

The lineage depleted cells were stained with following fluorophore-conjugated monoclonal antibodies: Cd105-PE, clone MJ7/18, Miltenyi; Cd4-Vioblue, clone REA604, Miltenyi; Cd11bVioblue, clone REA592, Miltenyi; Cd117-Pe Vio770, clone REA791, Miltenyi; Cd8a-Vioblue, clone 53-6.7, Miltenyi; CD50-Vioblue, clone REA421, Miltenyi; CD45R-Vioblue, clone REA755, Miltenyi; GR1-Vioblue, clone REA810, Miltenyi; Sca-APC, clone REA422, Miltenyi; CD48-APC Cy7, clone HM48-1, Miltenyi; CD150-BV510, clone TC15-12F12, CD34-PeCy5, MEC147, Miltenyi. Approximately 10,000 LK (Lin-, CD117+) cells per sample were sorted using the BD FACSMelody cell sorter (BD Biosciences, San Jose, California) into $1 \times$ PBS containing 4\% BSA. For low input and single-cell $\mathrm{qPCR}$, a pool of 25 cells and single LSK CD150+ CD48- CD34- HSCs respectively were sorted directly into Smart-seq2 lysis buffer ${ }^{42}$.

\section{Sequencing of single-cell cDNA libraries}

Sorted cells were processed by 3' end single cell RNA-Seq using the 10X Genomics Chromium (V2 Kit) according to manufacturer's protocol (10X Genomics, Pleasanton, CA) with an increase to 16 cycles for the CDNA PCR amplification. Libraries were sequenced on a NextSeq 500 or NovaSeq 6000 (Illumina, San Diego) in paired end, single index mode as per the $10 X$ Genomics recommended metrics.

Raw Illumina sequencing data were analysed with the 10X Genomics CellRanger pipeline (version 3.0.2) to obtain a single-cell expression matrix object. Subsequent analysis was performed in R using Seurat version $3^{16}$. Cells showing gene counts lower than 1,000 and a mitochondrial gene expression percentage higher than $5 \%$ were excluded from further analysis. Within Seurat, data were normalised using NormalizeData (normalization.method = 
"LogNormalize", scale.factor $=10000$ ) and data from multiple samples were merged using the FindIntegrationAnchors and IntegrateData commands.

\section{Pacific Biosciences Sequel Sequencing of single-cell cDNA libraries}

Libraries compatible for the Pacific Biosciences Sequel/Sequel II systems were prepared from 800 ng input cDNA, following the "no size selection" Iso-Seq library preparation method according to the manufacturer's instructions (IsoSeq Template Preparation for Sequel System V05), with the following modifications: the elution incubation time during AMPure beads purification was increased to 10 minutes and the second AMPure bead purification step, following the exonuclease reaction, was omitted to optimise library concentration.

\section{Pacific Biosystems long-read analysis}

Circular Consensus reads (CCS) were generated using the following parameters: maximum subread length 20,000 , minimum subread length 50 and minimum number of passes 3 . Reads with identified polydA or polydT were demultiplexed using bbduk https://sourceforge.net/projects/bbmap/) $(\mathrm{k}=16$, hdist $=3)$ using the 10X genomics barcodes identified from the short-read analysis. Long-reads were mapped to the mouse genome (mm10) using Minimap 2 (v2.17), and to the gencode (vM19) transcriptome.

Novel exons were identified by investigating the alignment of the reads to the transcriptome identifying inserts of at least 21 nucleotides located at exon junctions. To confirm the existence of these exons, the alignment of the reads to the genome was parsed, with exonic sequences located within the previously identified intron, and supported by at least two reads retained for further analysis. We further removed any exonic regions overlapping RefSeq annotations (GRCm38, last accessed February 19 2019).

To identify reads supporting $\mathrm{V}(\mathrm{D}) \mathrm{J}$ recombination events, we used IgBlast $\mathrm{v} 1.14 .0{ }^{43}$ ) using default parameters (-min_V_length 9 -min_J_length 0 -min_D_match 5 -D_penalty -2 J_penalty -2). 


\section{Custom transcriptome annotation}

A technology-agnostic long-read pipeline TALON v.5.0 [1], was used to construct a custom transcriptome annotation. First, 11 genome-aligned sam files were passed to TranscriptClean v.2.0.2 [2] for correction of read microindels $(<5 \mathrm{bp})$ and mismatches, though any noncanonical splice junctions were retained for downstream analyses and clean-up was not variant-aware. Internal priming artifacts are a known issue with oligo-dT selection methods [3] and this was assessed using a T-window size of $20 \mathrm{bp}$ (equivalent to the primer T sequence), with reads labelled accordingly using TALON functions. Read annotation was performed using the Mus musculus Gencode v.M24 reference annotation gtf and GRCm38.p6 genome with minimum alignment identity $=0.9$ and coverage $=0.8$ on the samples. Identified transcripts were subsequently filtered using a minimum count threshold of $\mathrm{N}=5$ reads in $\mathrm{K}=3$ samples. Thresholds were selected to balance sensitivity with accuracy. An updated annotation was produced using this filtered set of transcripts. Novel antisense transcripts that perfectly matched existing gene models were removed as unreliable mappings. The TALON custom gtf contains only features detected with reads present in the dataset, so a complete custom transcriptome annotation was compiled by merging the Gencode v.M24 reference and custom TALON gtfs. Transcript coding potential was assessed through frame preservation and applying CPAT2.0 ${ }^{44}$ using Gencode long non coding RNAs (https://www.gencodegenes.org/) and CDS as training sets.

\section{Data integration in Seurat}

In order to reduce the batch effect, count matrices produced by short-read sequencing for individual libraries were combined in Seurat using FindIntegrationAnchors and IntegrateData functions (dims $=1: 20$ ). Illumina and PacBio reads were integrated in Seurat using the CreateAssayObject command to add the long-read data to an existing Seurat object already containing the short-read data. This links the demultiplexed long-reads with the short-read data through the cell barcodes present in both.

\section{Single-cell and low-input qPCR}

Amplified cDNA was generated from sorted cells using the Smart-seq2 protocol ${ }^{42}$. This material was then used as input for qPCR reactions using assays targeting Sult1a1 and Nupr1. Low-volume qPCR reactions were set up using the Mosquito HV instrument (STP Labtech) and analysed using LightCycler (Roche). For relative abundance, data are presented as expression relative to a housekeeping gene. For single-cell isoform junction detection PCRs, 
reactions were performed as above using junction-spanning primers. Data are presented as presence/absence heatmap, where analyses with $\mathrm{Ct}$ values $<30$ were considered to be expressed.

Data availability

The raw sequencing data can be accessed on the NCBI-SRA archive, accession number PRJNA691569. 


\section{Figure Legends}

Figure 1
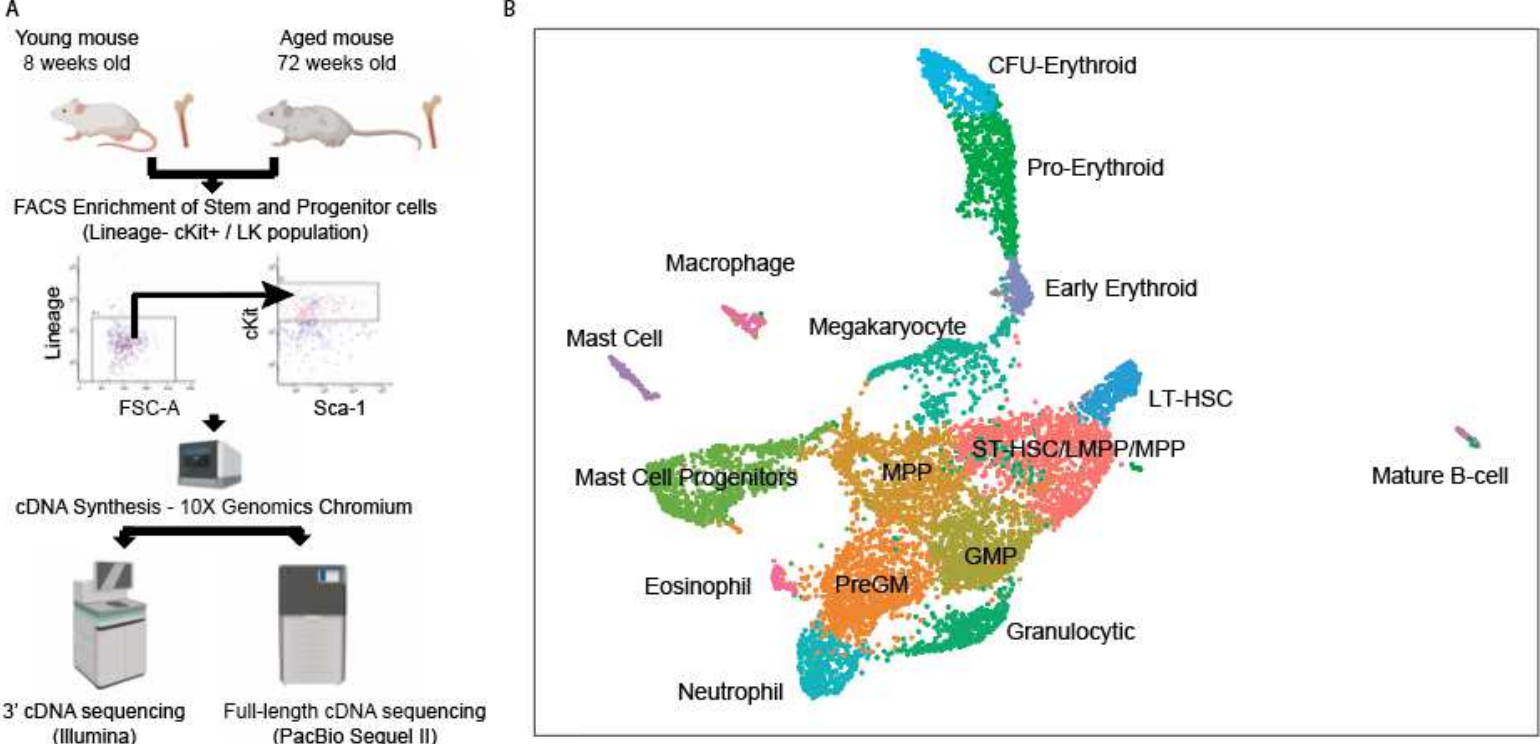

Figure 1: Profiling changes in cellular composition and gene expression in ageing haematopoiesis. A) Experimental overview. Following sorting, Lin- cKit+ cells were analysed by parallel short- and long-read scRNA-seq. B) Cell clustering revealed cellular heterogeneity in the Lin- cKit+ cell population including all myeloid progenitor cell types. 


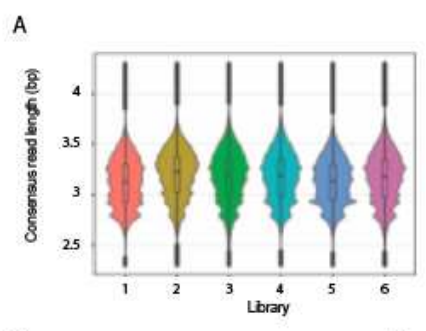

B

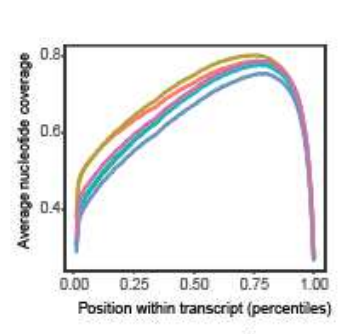

H

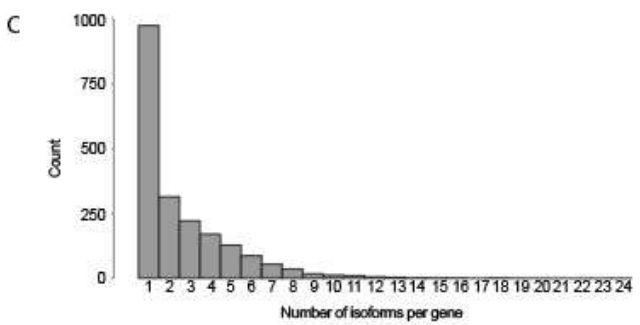

D

E
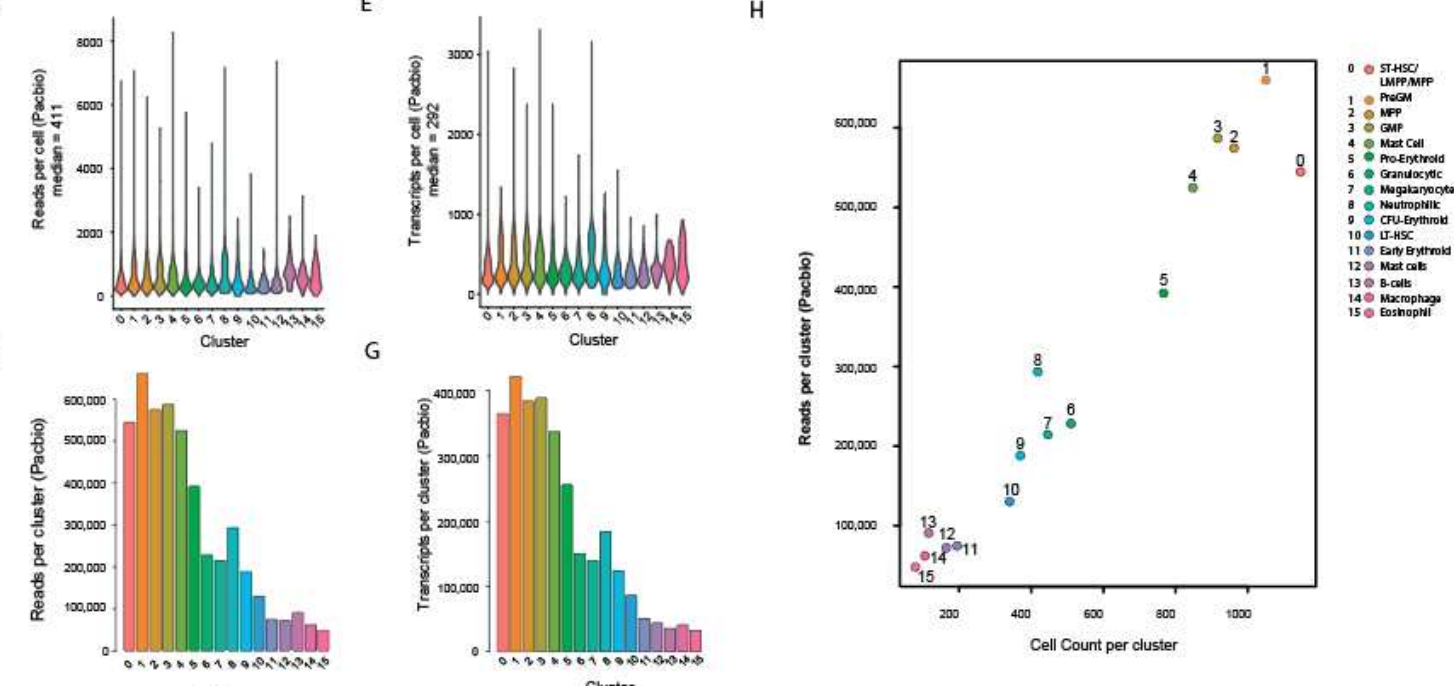

Cliliter

Cluster

Figure 2: Long-read sequencing from 10X Genomics Chromium cDNA. A) Read length distribution from long-read sequencing of 10X single-cell libraries. B) transcript coverage from long-read sequencing of $10 \mathrm{X}$ single-cell libraries. C) Number of isoforms detected per gene. D) Number of long-reads per cell, separated by cluster (as identified in Fig 1B) E) Number of transcripts per cell, again separated by cluster F) Total number of long-reads per cluster $\mathbf{G}$ ) Total number of transcripts per cluster. $\mathbf{H}$ ) Correlation of number of long-reads per cluster with cluster cell number. 


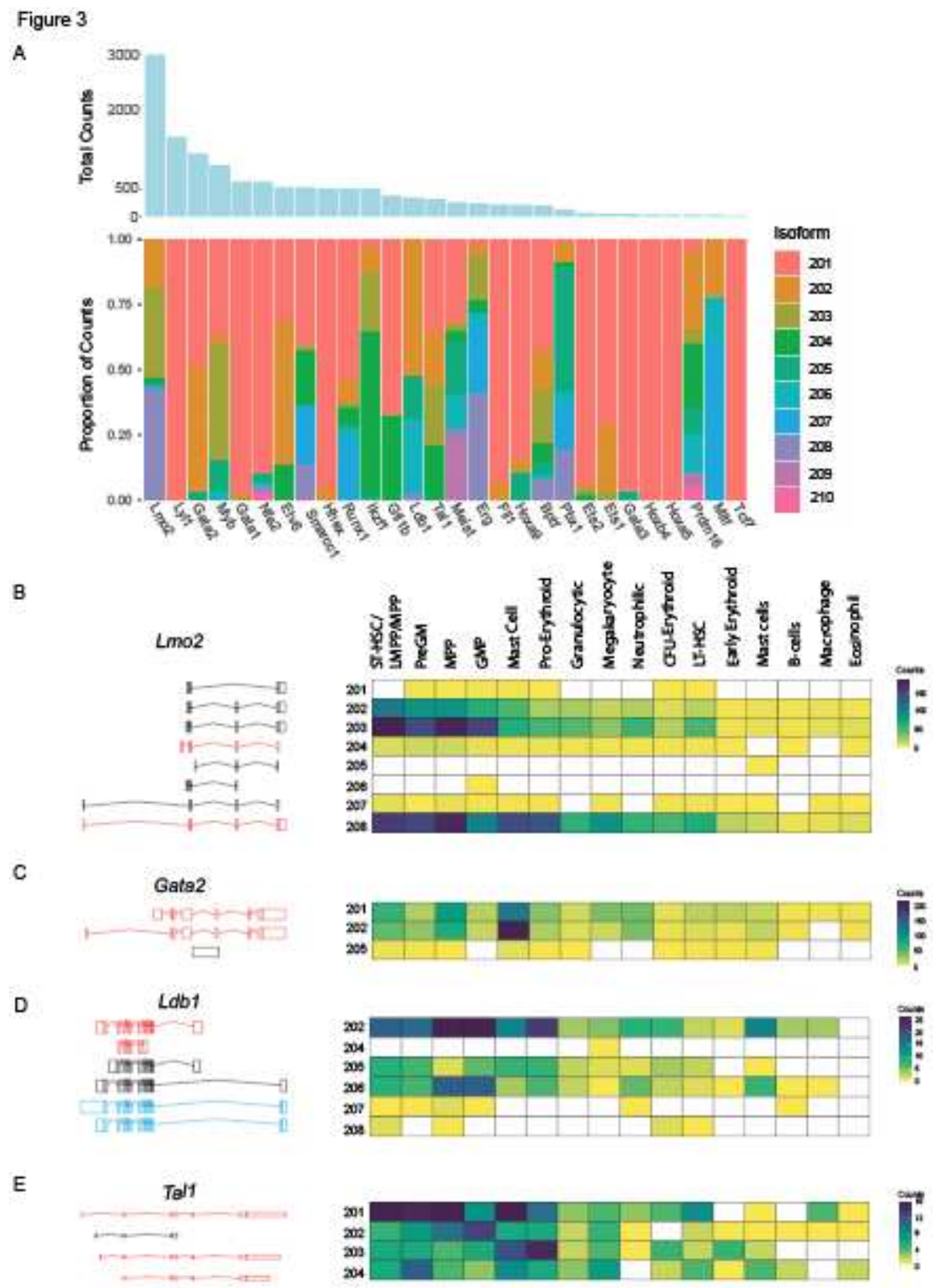

Figure 3: Heterogeneity in hematopoietic transcription factor isoform expression.

A) Total long-read counts and isoform frequency plots for a set of transcription factors implicated in hematopoiesis. B-D) Cell-type expression of isoforms of Lmo2, Gata2, Ldb1 and Tal1. Transcripts colored red or blue indicate sets of transcripts which encode the same protein, those in black encode distinct proteins from the same gene. 


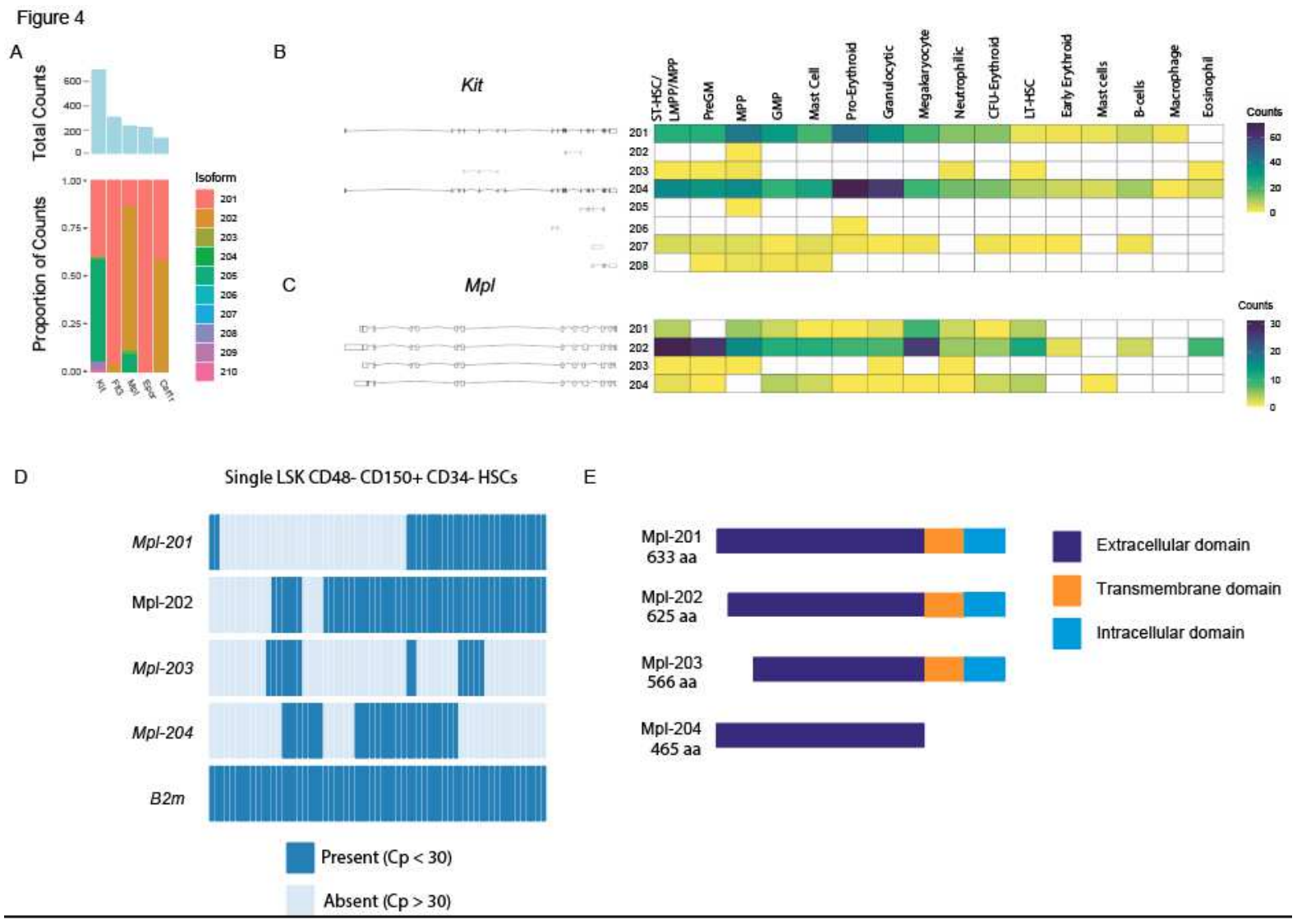

Figure 4: Heterogeneity in hematopoietic cytokine receptor isoform expression A) Total long-read counts and isoform frequency plots for key hematopoietic cytokine receptors B-C) Cell-type expression of isoforms of Kit and $\mathrm{Mpl}$. Transcripts in black encode distinct proteins from the same gene. Those in grey are non-coding. D) Single-cell isoform detection in $\mathrm{Mp} /$ by junction qPCR. Heatmap showing isoform expression in individual cells (columns) of four isoforms of $\mathrm{Mpl}$. B2m is presented as a positive control. E) Diagram of the distinct protein structures encoded by the distinct isoforms of $\mathrm{Mpl}$. 


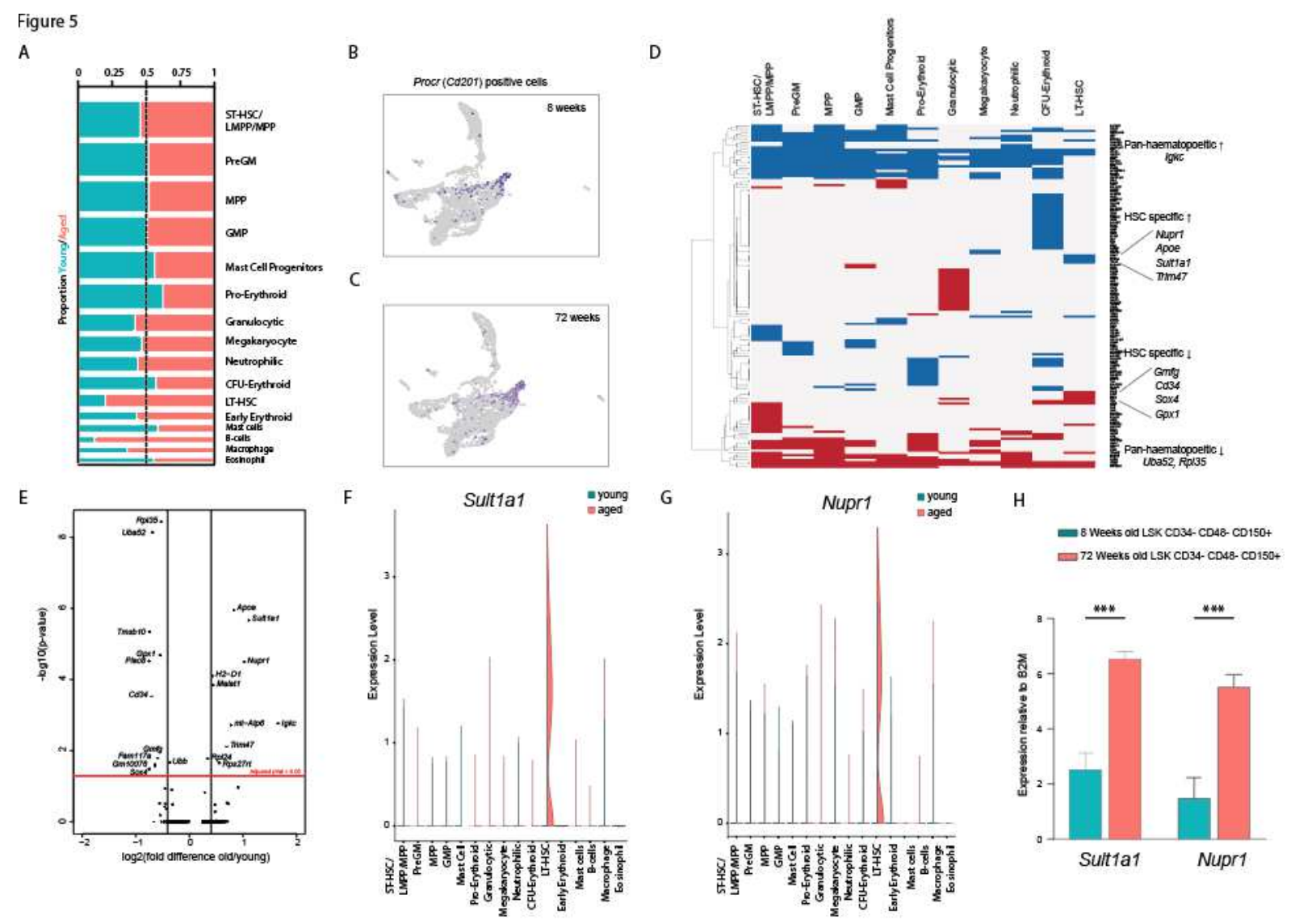

Figure 5: Cell frequency and gene expression changes in aged hematopoiesis A) Frequency of cells per cluster between young and aged mice. B-C) Procr expressing cells (HSCs) in young and aged mice, respectively. D) Differential expression for each cluster, based on short read data. Data are presented as a heatmap of fold-change values, where blue is upregulated with age and red is downregulated with age. E) Differential expression in the LT-HSC cluster. F) Expression of Sult1a1 in all clusters young and aged mice from shortread data G) Expression of Nupr1 in all clusters in young and aged mice from short-read data. H) qPCR confirmation of Sult1a1 and Nupr1 upregulation in aged phenotypic stem cells (*** indicates $p$-value $<0.001)$ 
A

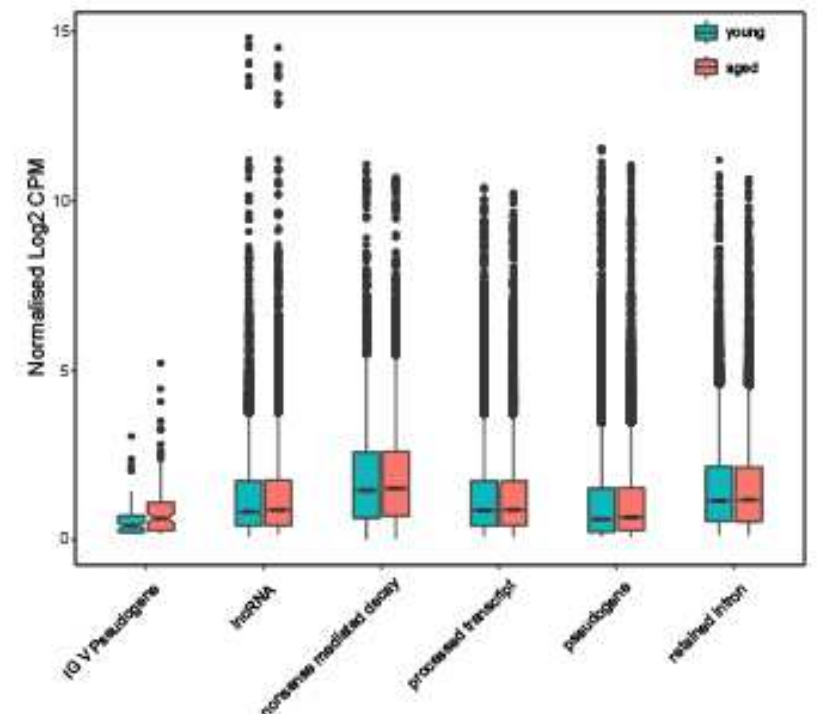

B Igkc-short read

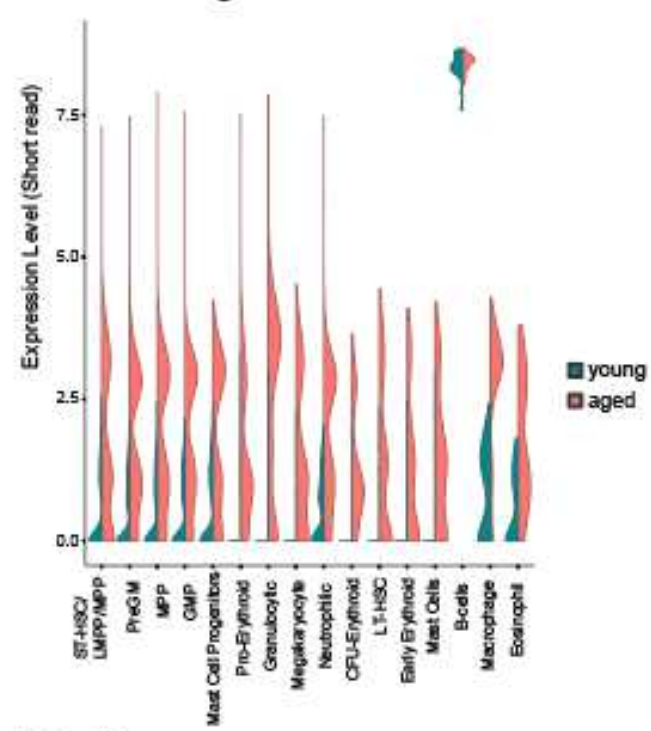

C

lgk - long read (HSC derived)

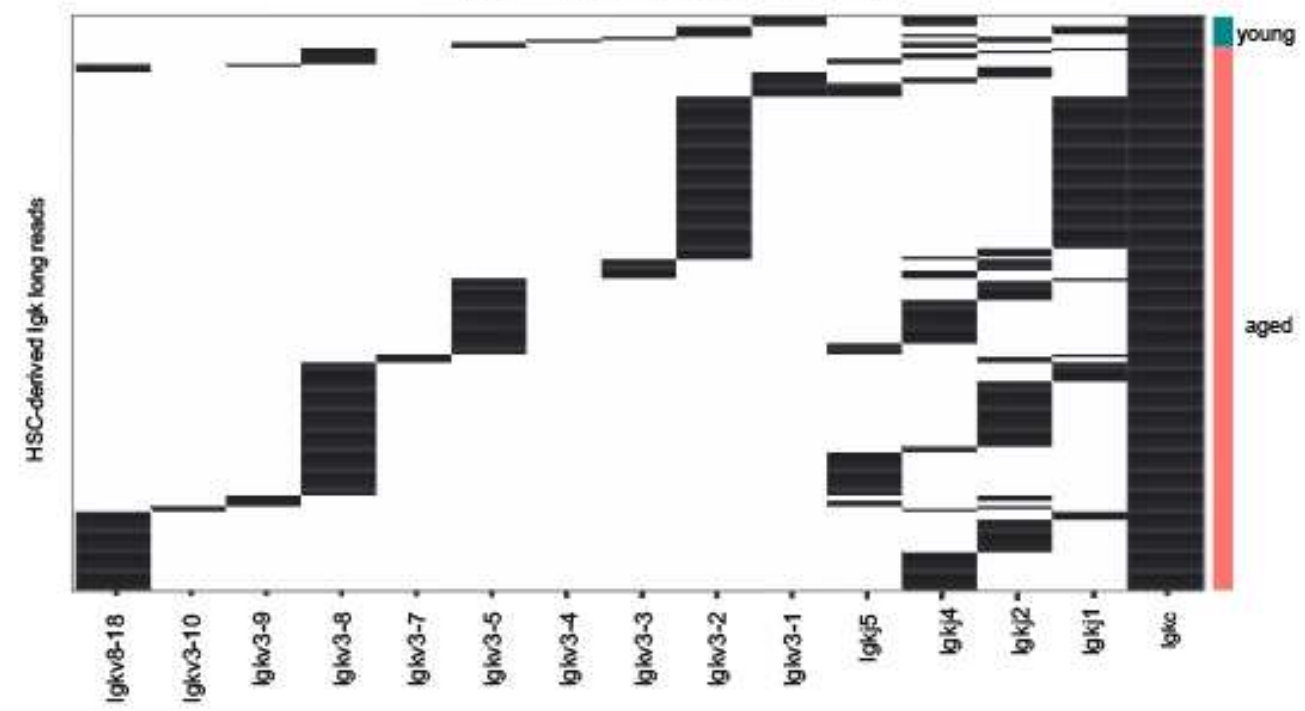

Figure 6: Integration of long- and short- read data to profile cell type isoform usage. Changes in classes of transcript between old and young mice, based on the long-read sequencing data B) Expression of Igkc from young and aged mice in all clusters from shortread data. C) Observation of full length, VJ recombined lgk transcripts in long-reads derived from the HSC cluster. Data are shown as a heatmap of long-reads (rows) with presence of V and $\mathrm{J}$ regions (columns) indicated in black. 


\section{Supplementary Figures}

Supplementary Figure 1

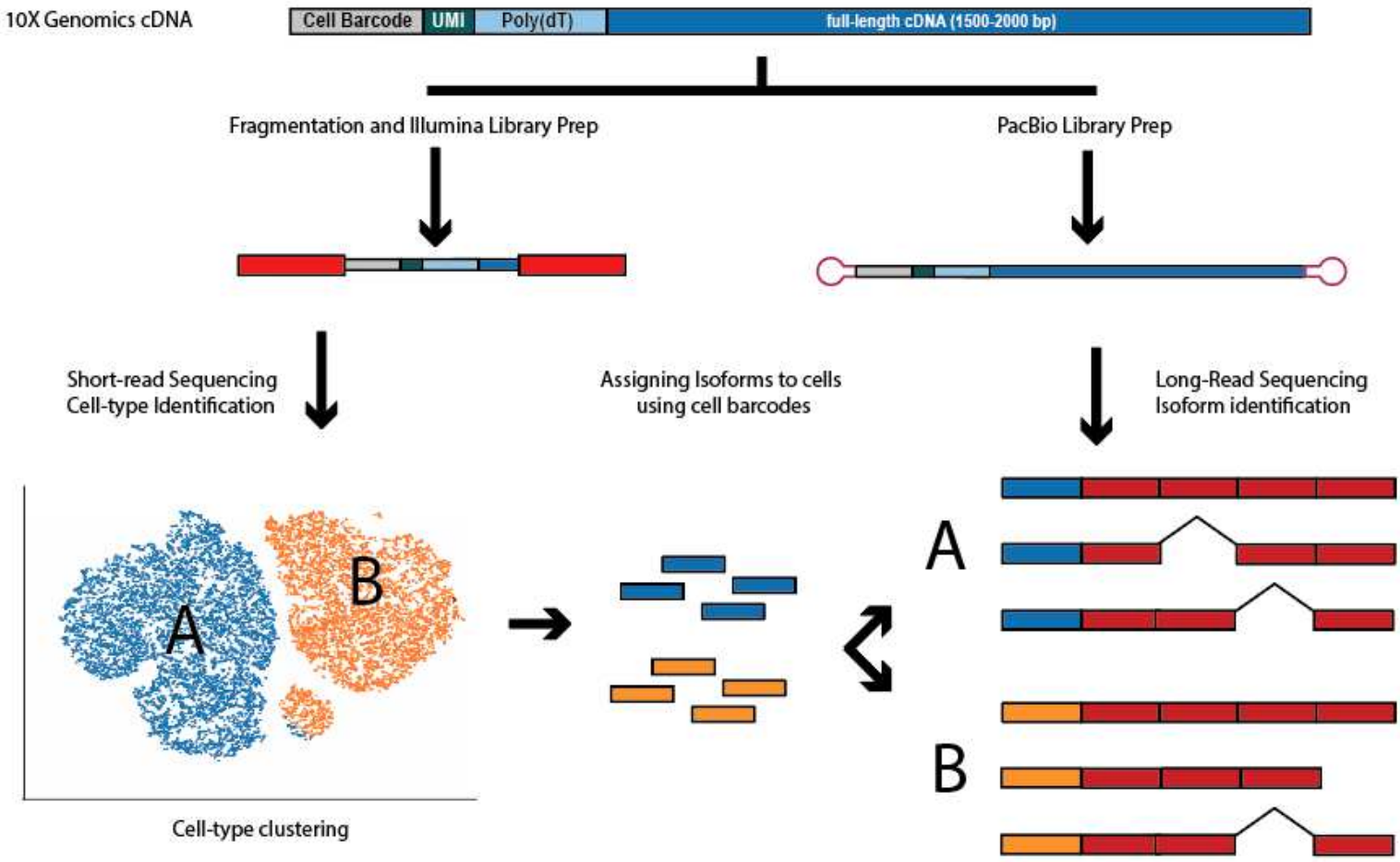

Supplementary Figure 1: Experimental overview. Full-length cDNA is generated by the 10X Genomics Chromium instrument. This material is used as input for conventional shortread (Illumina) and long-read (PacBio) sequencing, which enables sequencing of the fulllength of the transcript and detection of alternative splicing or recombination events. Cell clustering is performed on the short-read data. Integrating the cell barcodes present in both the short- and long-read data enables assignment of long-reads to cells and cell clusters. 


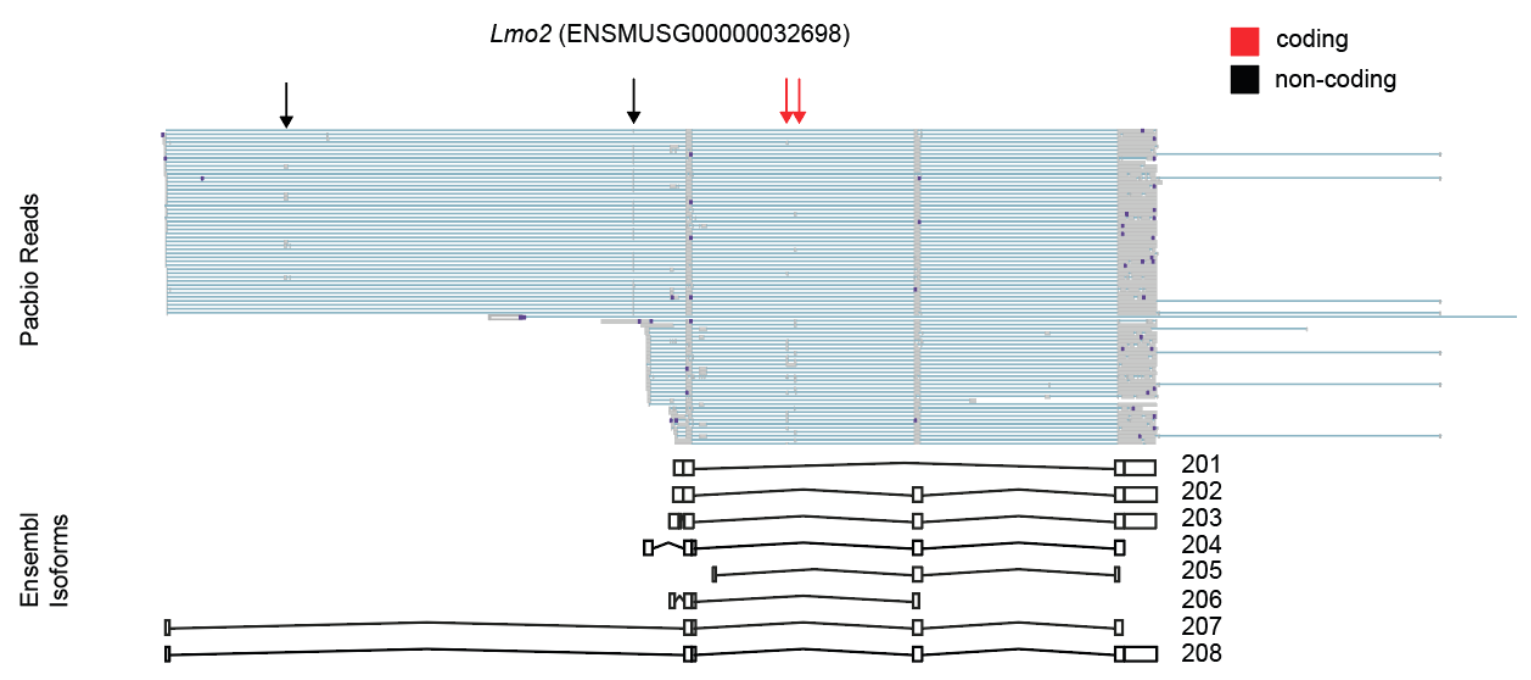

Supplementary Figure 2: Novel exon discovery in Lmo2. Representative Pacbio reads are shown above the Ensembl annotated transcripts for $L$ mo2. Multiple novel in frame exons were observed in the long-read data, indicated by arrows, including two which generates isoforms of the transcript which would encode a distinct protein sequences (highlighted in red). 


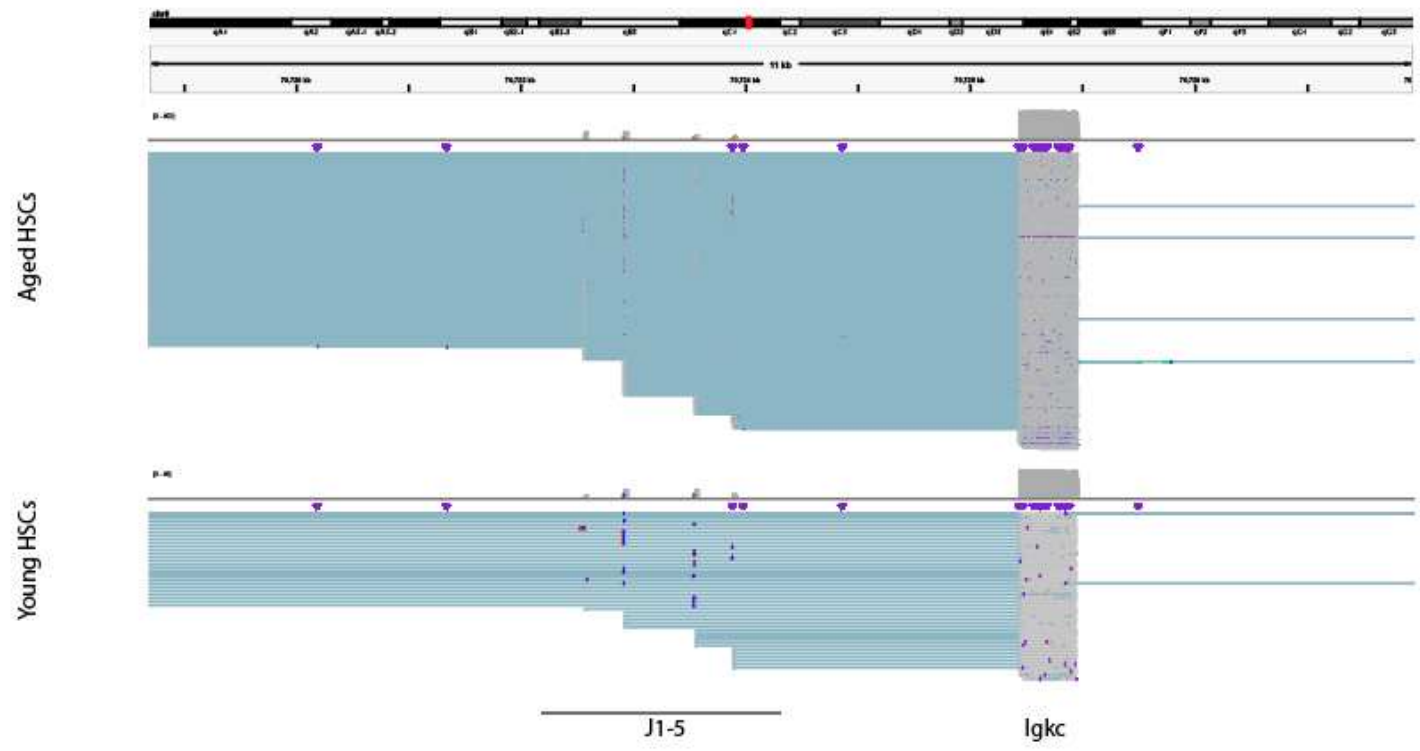

Supplementary Figure 3: Reads from the Immunoglobulin kappa locus. Reads from young and aged LT-HSCs visualised in the Integrated Genome Viewer (IGV) show recombination between the Igkc locus and the $\mathrm{J}$ domains. 


\section{Supplementary Tables}

Supplementary Table 1: Marker genes for clusters (relating to Figure 1B)

Supplementary Table 2: Long-read sequencing statistics (relating to Figure 2)

Supplementary Table 3: Novel exons detected in long-read sequencing data.

Supplementary table 4 : Differential gene expression between young and aged HSCs (relating to Figure 5E) 


\section{References}

1. Svensson, V., Vento-Tormo, R. \& Teichmann, S. A. Exponential scaling of single-cell RNA-seq in the past decade. Nat. Protoc. 13, 599-604 (2018).

2. Dahlin, J. S. et al. A single-cell hematopoietic landscape resolves 8 lineage trajectories and defects in Kit mutant mice. Blood 131, e1-e11 (2018).

3. Pijuan-Sala, B. et al. A single-cell molecular map of mouse gastrulation and early organogenesis. Nature 566, 490-495 (2019).

4. Tabula Muris Consortium et al. Single-cell transcriptomics of 20 mouse organs creates a Tabula Muris. Nature 562, 367-372 (2018).

5. Laurenti, E. \& Göttgens, B. From haematopoietic stem cells to complex differentiation landscapes. Nature 553, 418-426 (2018).

6. Frankish, A. et al. GENCODE reference annotation for the human and mouse genomes. Nucleic Acids Res. 47, D766-D773 (2019).

7. Pan, Q., Shai, O., Lee, L. J., Frey, B. J. \& Blencowe, B. J. Deep surveying of alternative splicing complexity in the human transcriptome by high-throughput sequencing. Nat. Genet. 40, 1413-1415 (2008).

8. Marti-Solano, M. et al. Combinatorial expression of GPCR isoforms affects signalling and drug responses. Nature (2020) doi:10.1038/s41586-020-2888-2.

9. Goldstein, O. et al. Mapping Whole-Transcriptome Splicing in Mouse Hematopoietic Stem Cells. Stem Cell Reports 8, 163-176 (2017).

10. Chen, L. et al. Transcriptional diversity during lineage commitment of human blood progenitors. Science 345, 1251033 (2014).

11. Hardwick, S. A., Joglekar, A., Flicek, P., Frankish, A. \& Tilgner, H. U. Getting the Entire Message: Progress in Isoform Sequencing. (2019) doi:10.3389/fgene.2019.00709.

12. Pronk, C. J. H. et al. Elucidation of the phenotypic, functional, and molecular topography of a myeloerythroid progenitor cell hierarchy. Cell Stem Cell 1, 428-442 (2007).

13. Balazs, A. B., Fabian, A. J., Esmon, C. T. \& Mulligan, R. C. Endothelial protein C 
receptor (CD201) explicitly identifies hematopoietic stem cells in murine bone marrow. Blood 107, 2317-2321 (2006).

14. Gupta, I. et al. Single-cell isoform RNA sequencing characterizes isoforms in thousands of cerebellar cells. Nat. Biotechnol. (2018) doi:10.1038/nbt.4259.

15. Wyman, D. et al. A technology-agnostic long-read analysis pipeline for transcriptome discovery and quantification. bioRxiv 672931 (2019) doi:10.1101/672931.

16. Butler, A., Hoffman, P., Smibert, P., Papalexi, E. \& Satija, R. Integrating single-cell transcriptomic data across different conditions, technologies, and species. Nat. Biotechnol. 36, 411-420 (2018).

17. Hamey, F. K. et al. Reconstructing blood stem cell regulatory network models from single-cell molecular profiles. Proc. Natl. Acad. Sci. U. S. A. 114, 5822-5829 (2017).

18. Sun, W. et al. Homo-binding character of LMO2 isoforms and their both synergic and antagonistic functions in regulating hematopoietic-related target genes. J. Biomed. Sci. $17,22(2010)$.

19. Rodrigues, N. P., Tipping, A. J., Wang, Z. \& Enver, T. GATA-2 mediated regulation of normal hematopoietic stem/progenitor cell function, myelodysplasia and myeloid leukemia. Int. J. Biochem. Cell Biol. 44, 457-460 (2012).

20. Montero, J. C., López-Pérez, R., San Miguel, J. F. \& Pandiella, A. Expression of c-Kit isoforms in multiple myeloma: differences in signaling and drug sensitivity. Haematologica 93, 851-859 (2008).

21. Lebedev, T. D. et al. Two Receptors, Two Isoforms, Two Cancers: Comprehensive Analysis of KIT and TrkA Expression in Neuroblastoma and Acute Myeloid Leukemia. Front. Oncol. 9, 1046 (2019).

22. Young, S. M., Cambareri, A. C., Odell, A., Geary, S. M. \& Ashman, L. K. Early myeloid cells expressing c-KIT isoforms differ in signal transduction, survival and chemotactic responses to Stem Cell Factor. Cell. Signal. 19, 2572-2581 (2007).

23. Coers, J., Ranft, C. \& Skoda, R. C. A truncated isoform of c-Mpl with an essential Cterminal peptide targets the full-length receptor for degradation. J. Biol. Chem. 279, 
36397-36404 (2004).

24. Dykstra, B. et al. Long-term propagation of distinct hematopoietic differentiation programs in vivo. Cell Stem Cell 1, 218-229 (2007).

25. Peters, M. J. et al. The transcriptional landscape of age in human peripheral blood. Nat. Commun. 6, 8570 (2015).

26. Kobayashi, M. et al. The ubiquitin hybrid gene UBA52 regulates ubiquitination of ribosome and sustains embryonic development. Sci. Rep. 6, 36780 (2016).

27. Kevei, É. \& Hoppe, T. Ubiquitin sets the timer: impacts on aging and longevity. Nat. Struct. Mol. Biol. 21, 290-292 (2014).

28. Adusumalli, S., Ngian, Z.-K., Lin, W.-Q., Benoukraf, T. \& Ong, C.-T. Increased intron retention is a post-transcriptional signature associated with progressive aging and Alzheimer's disease. Aging Cell 18, e12928 (2019).

29. Ong, C.-T. \& Adusumalli, S. Increased intron retention is linked to Alzheimer's disease. Neural Regeneration Res. 15, 259-260 (2020).

30. Chambers, S. M. et al. Aging hematopoietic stem cells decline in function and exhibit epigenetic dysregulation. PLoS Biol. 5, e201 (2007).

31. Sun, D. et al. Epigenomic profiling of young and aged HSCs reveals concerted changes during aging that reinforce self-renewal. Cell Stem Cell 14, 673-688 (2014).

32. Gazit, R. et al. Fgd5 identifies hematopoietic stem cells in the murine bone marrow. J. Exp. Med. 211, 1315-1331 (2014).

33. Marttila, S., Chatsirisupachai, K., Palmer, D. \& de Magalhães, J. P. Ageing-associated changes in the expression of IncRNAs in human tissues reflect a transcriptional modulation in ageing pathways. Mech. Ageing Dev. 185, 111177 (2020).

34. Jin, L., Song, Q., Zhang, W., Geng, B. \& Cai, J. Roles of long noncoding RNAs in aging and aging complications. Biochim. Biophys. Acta Mol. Basis Dis. 1865, 1763-1771 (2019).

35. Sanjuan-Pla, A. et al. Platelet-biased stem cells reside at the apex of the haematopoietic stem-cell hierarchy. Nature 502, 232-236 (2013). 
36. Chen, Z., Qiu, X. \& Gu, J. Immunoglobulin expression in non-lymphoid lineage and neoplastic cells. Am. J. Pathol. 174, 1139-1148 (2009).

37. Huang, J. et al. Rearrangement and expression of the immunoglobulin $\mu$-chain gene in human myeloid cells. Cell. Mol. Immunol. 11, 94-104 (2014).

38. Fuchs, T. et al. Expression of combinatorial immunoglobulins in macrophages in the tumor microenvironment. PLoS One 13, e0204108 (2018).

39. Liu, J. et al. Immunoglobulin gene expression in umbilical cord blood-derived CD34+ hematopoietic stem/progenitor cells. Gene 575, 108-117 (2016).

40. Qiu, X. et al. Immunoglobulin gamma heavy chain gene with somatic hypermutation is frequently expressed in acute myeloid leukemia. Leukemia 27, 92-99 (2013).

41. Saez, B., Walter, M. J. \& Graubert, T. A. Splicing factor gene mutations in hematologic malignancies. Blood 129, 1260-1269 (2017).

42. Picelli, S. et al. Full-length RNA-seq from single cells using Smart-seq2. Nat. Protoc. 9, $171-181$ (2014).

43. Ye, J., Ma, N., Madden, T. L. \& Ostell, J. M. IgBLAST: an immunoglobulin variable domain sequence analysis tool. Nucleic Acids Res. 41, W34-40 (2013).

44. Wang, L. et al. CPAT: Coding-Potential Assessment Tool using an alignment-free logistic regression model. Nucleic Acids Res. 41, e74 (2013). 


\section{Figures}

A Young mouse

8 weeks old

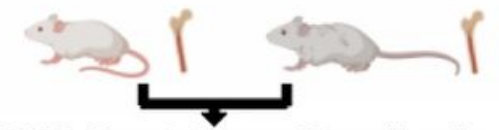

FACS Enrichment of Stem and Progenitor cells (Lineage-cKit+ / LK population)

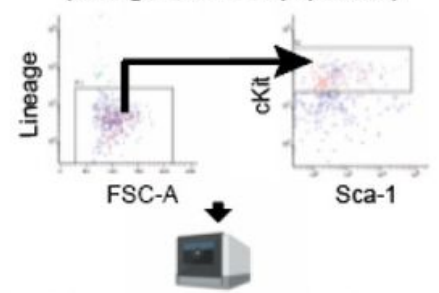

cDNA Synthesis - 10X Genomics Chromium

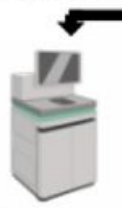

3' cDNA sequencing (Illumina)

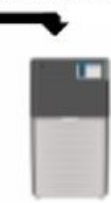

Full-length cDNA sequencing (PacBio Sequel II)
B

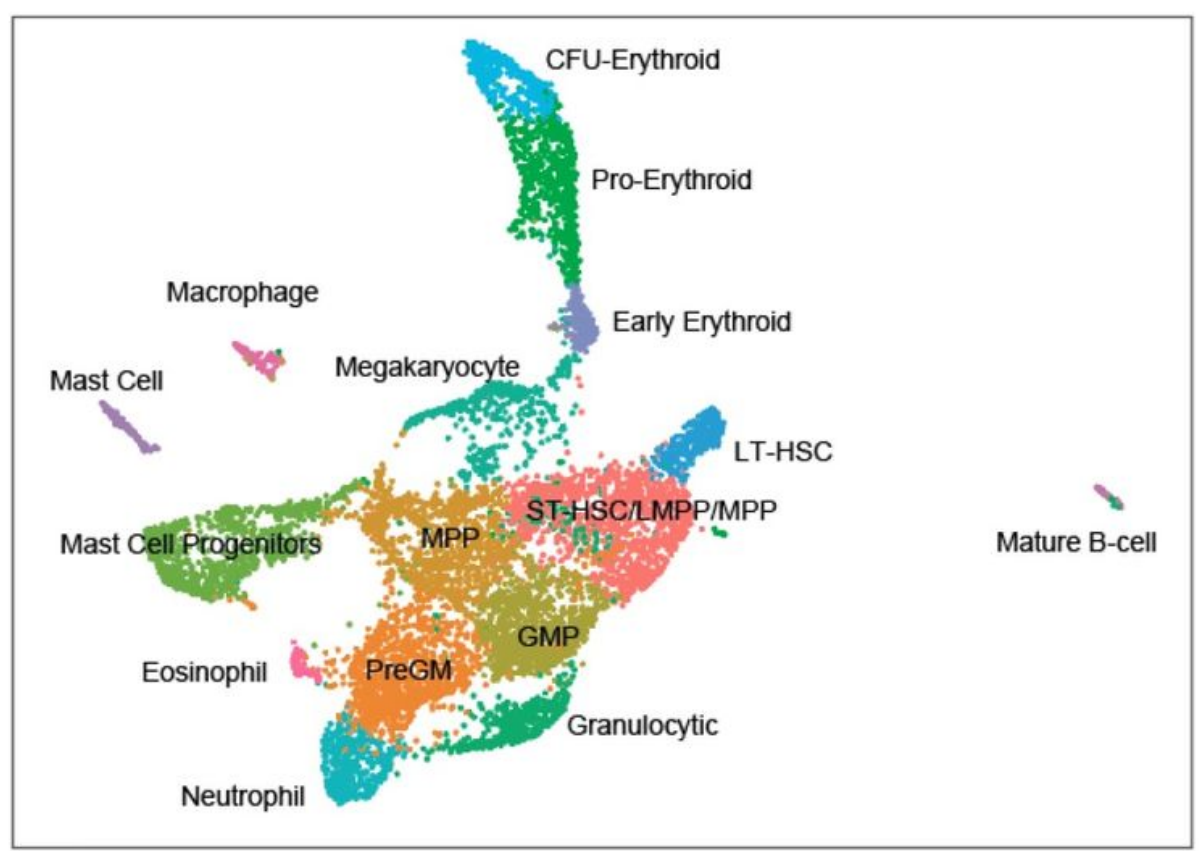

\section{Figure 1}

Profiling changes in cellular composition and gene expression in ageing haematopoiesis. A) Experimental overview. Following sorting, Lin- cKit+ cells were analysed by parallel short- and long-read scRNA-seq. B) Cell clustering revealed cellular heterogeneity in the Lin- cKit+ cell population including all myeloid progenitor cell types. 


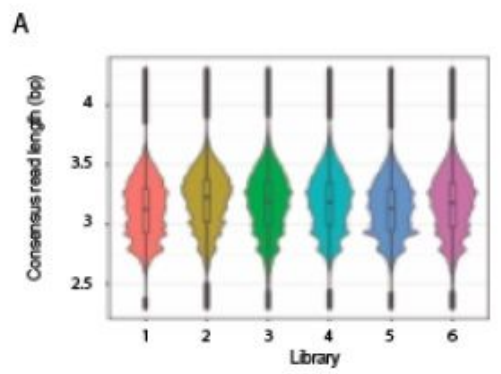

B

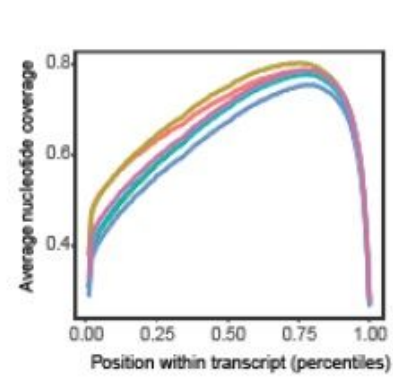

C

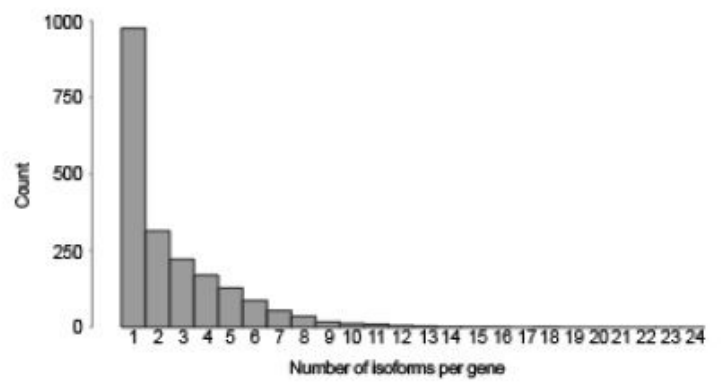

D

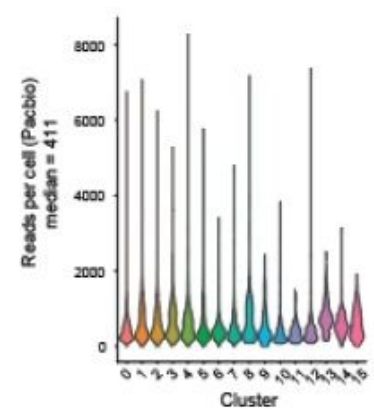

$\mathrm{F}$

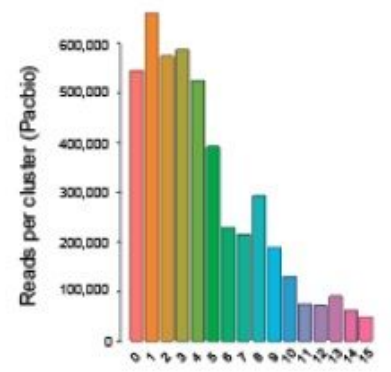

Cluster
E

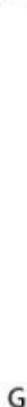

G
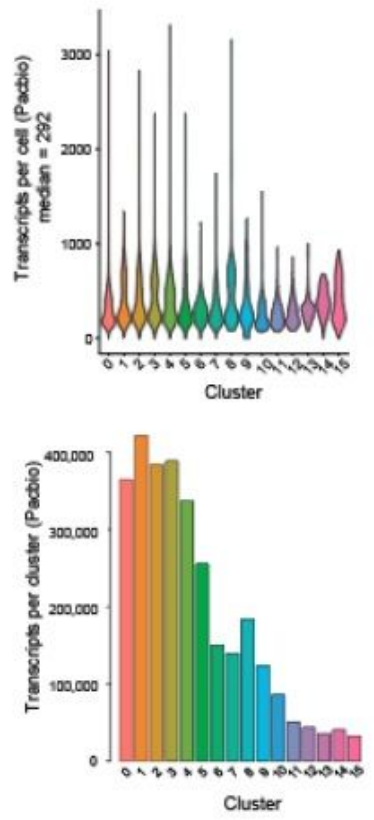

$\mathrm{H}$

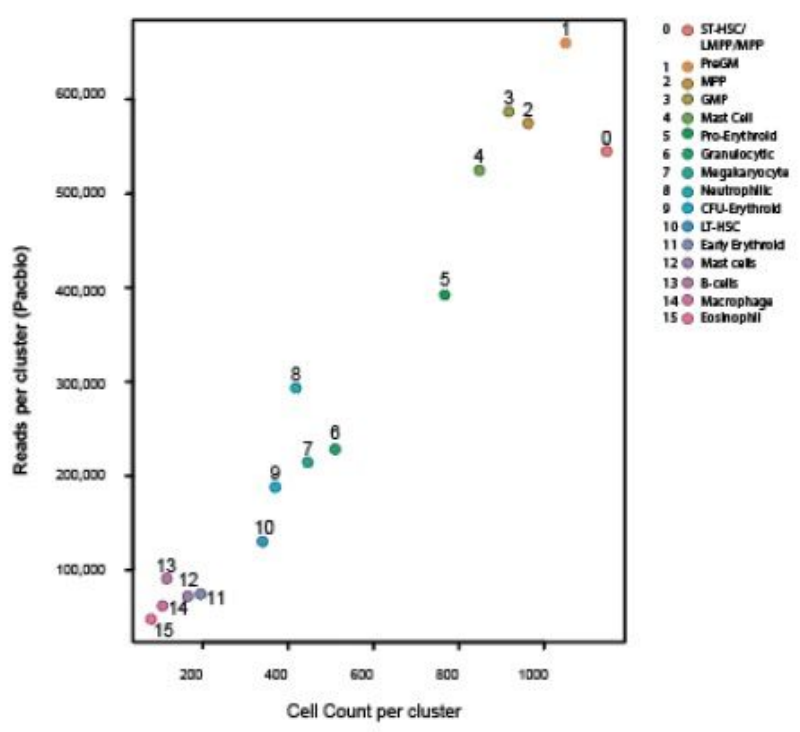

Figure 2

Long-read sequencing from 10X Genomics Chromium cDNA. A) Read length distribution from long-read sequencing of $10 \mathrm{X}$ single-cell libraries. $\mathrm{B}$ ) transcript coverage from long-read sequencing of $10 \mathrm{X}$ singlecell libraries. C) Number of isoforms detected per gene. D) Number of long-reads per cell, separated by cluster (as identified in Fig 1B) E) Number of transcripts per cell, again separated by cluster F) Total number of long-reads per cluster $G$ ) Total number of transcripts per cluster. $H$ ) Correlation of number of long-reads per cluster with cluster cell number. 
A

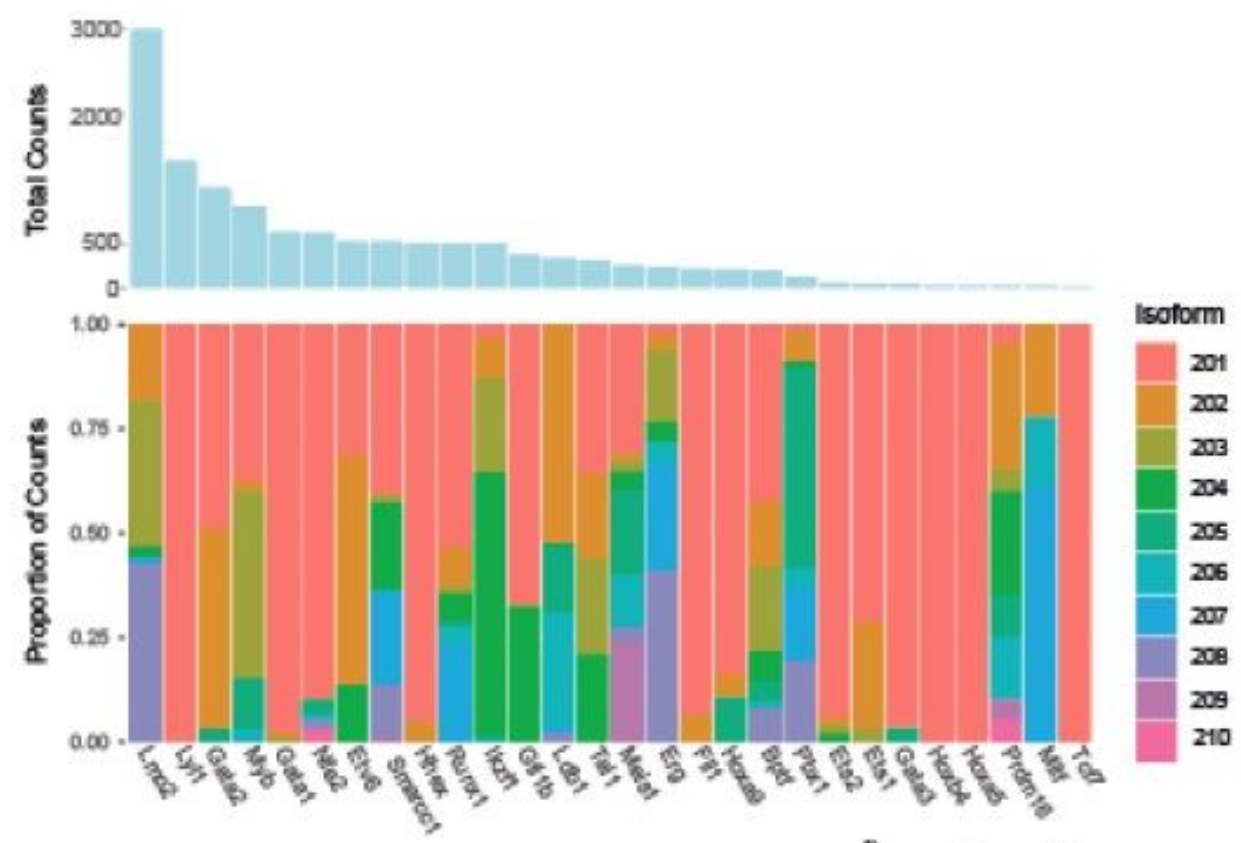

B

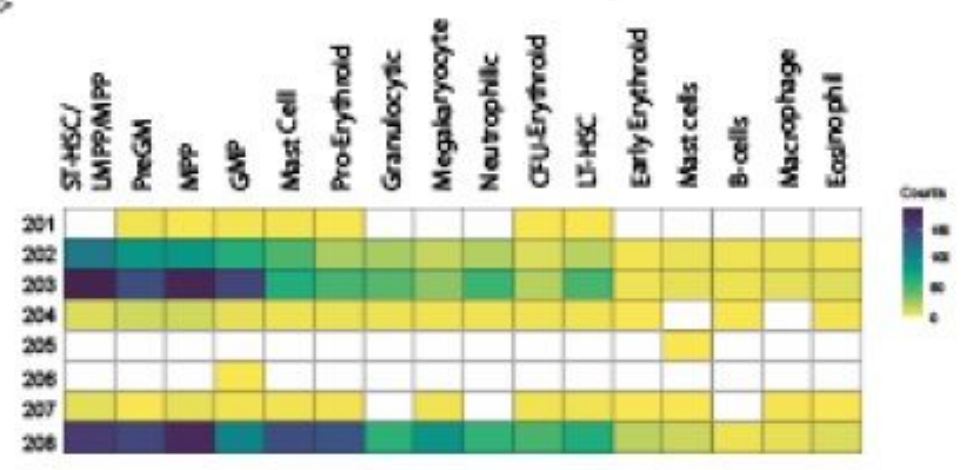

C
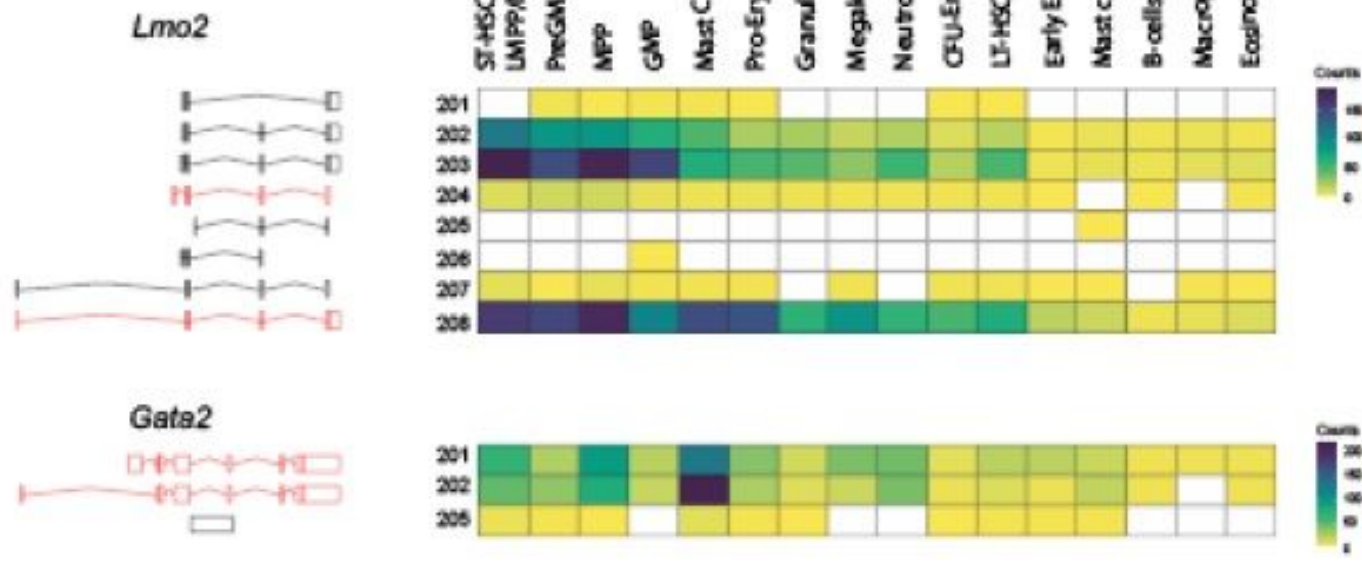

D

Lab1
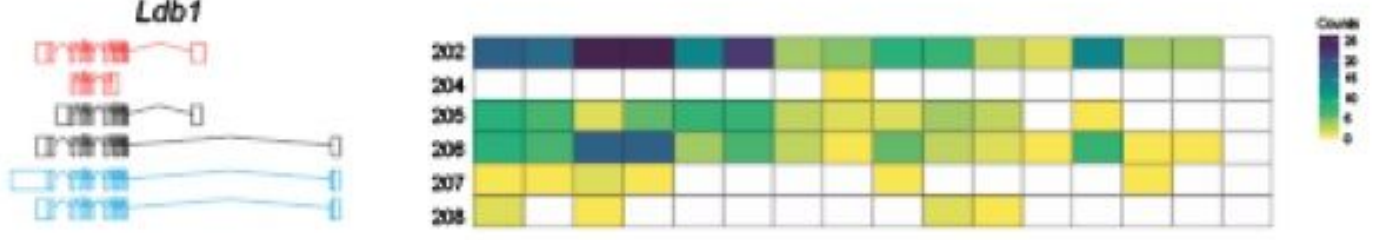

E
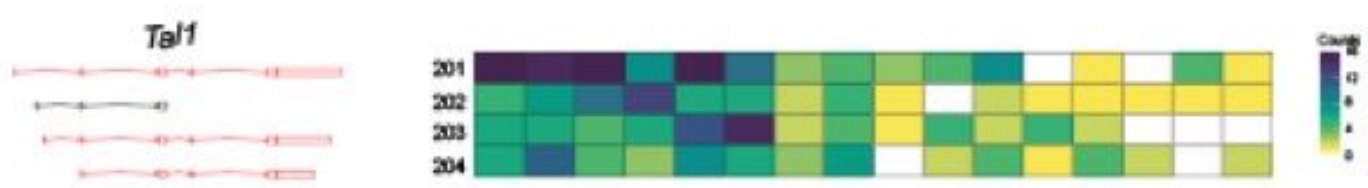

\section{Figure 3}

Heterogeneity in hematopoietic transcription factor isoform expression. A) Total long-read counts and isoform frequency plots for a set of transcription factors implicated in hematopoiesis. B-D) Cell-type expression of isoforms of Lmo2, Gata2, Ldb1 and Tal1. Transcripts colored red or blue indicate sets of transcripts which encode the same protein, those in black encode distinct proteins from the same gene. 
A

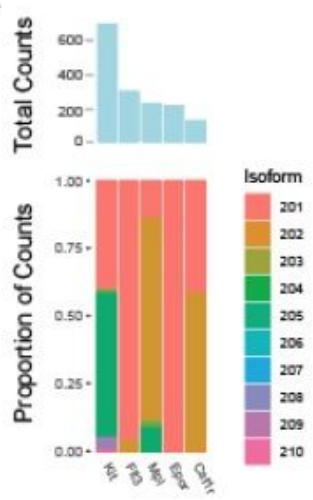

B

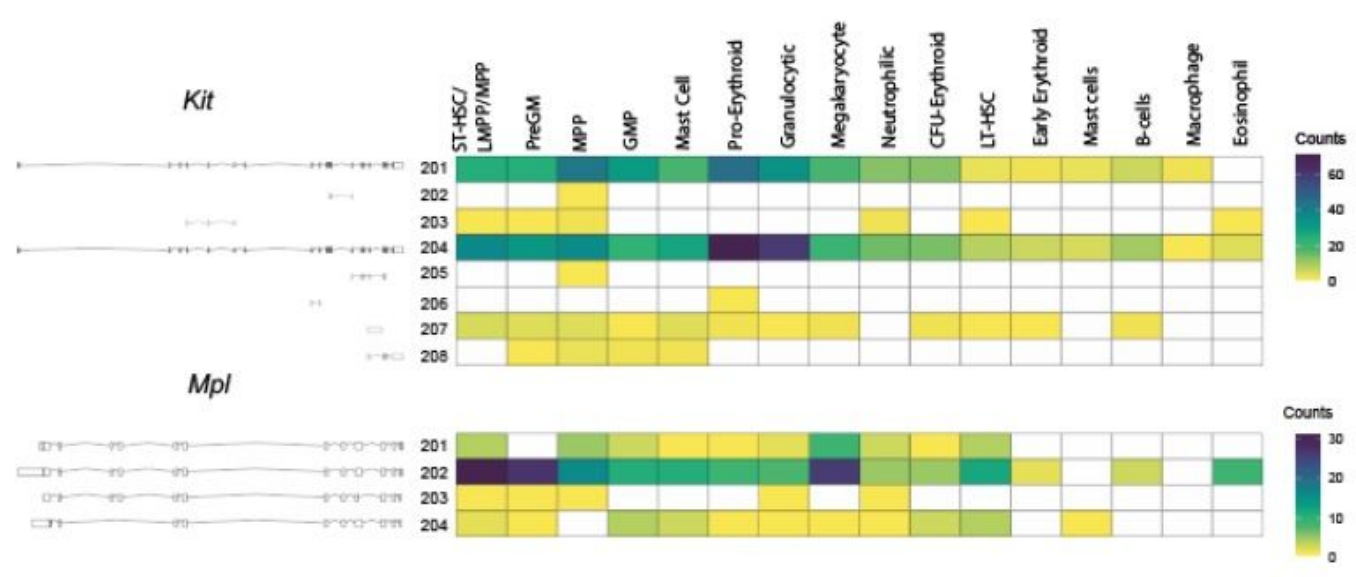

D

Single LSK CD48-CD150+ CD34- HSCs

$\mathrm{E}$

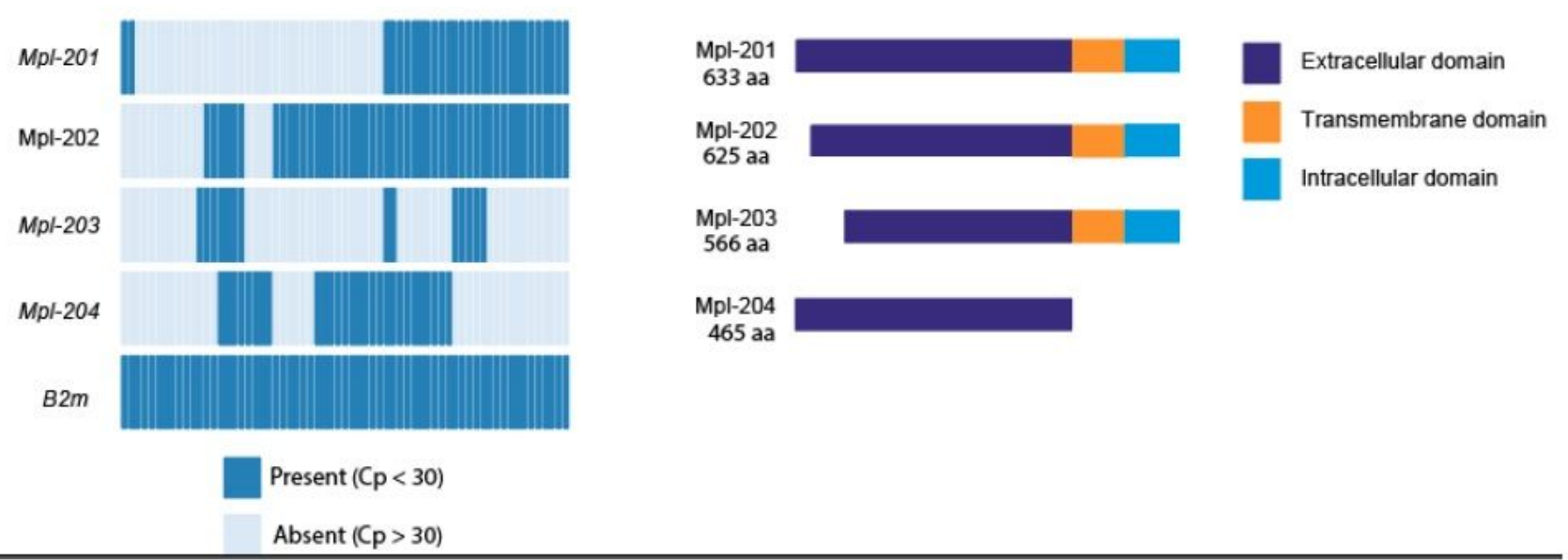

\section{Figure 4}

Heterogeneity in hematopoietic cytokine receptor isoform expression A) Total long-read counts and isoform frequency plots for key hematopoietic cytokine receptors B-C) Cell-type expression of isoforms of Kit and Mpl. Transcripts in black encode distinct proteins from the same gene. Those in grey are noncoding. D) Single-cell isoform detection in Mpl by junction qPCR. Heatmap showing isoform expression in individual cells (columns) of four isoforms of Mpl. B2m is presented as a positive control. E) Diagram of the distinct protein structures encoded by the distinct isoforms of Mpl. 
A

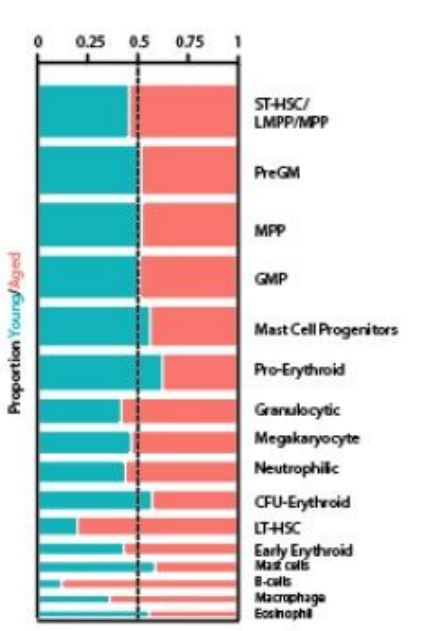

$E$

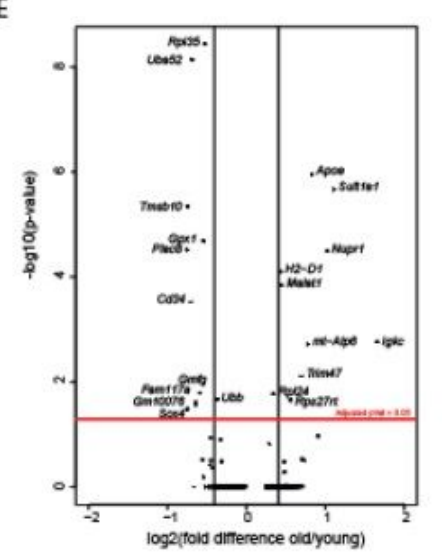

B

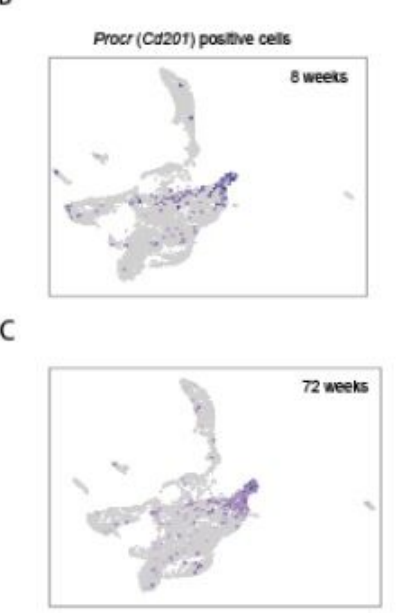

$\mathrm{F}$

D

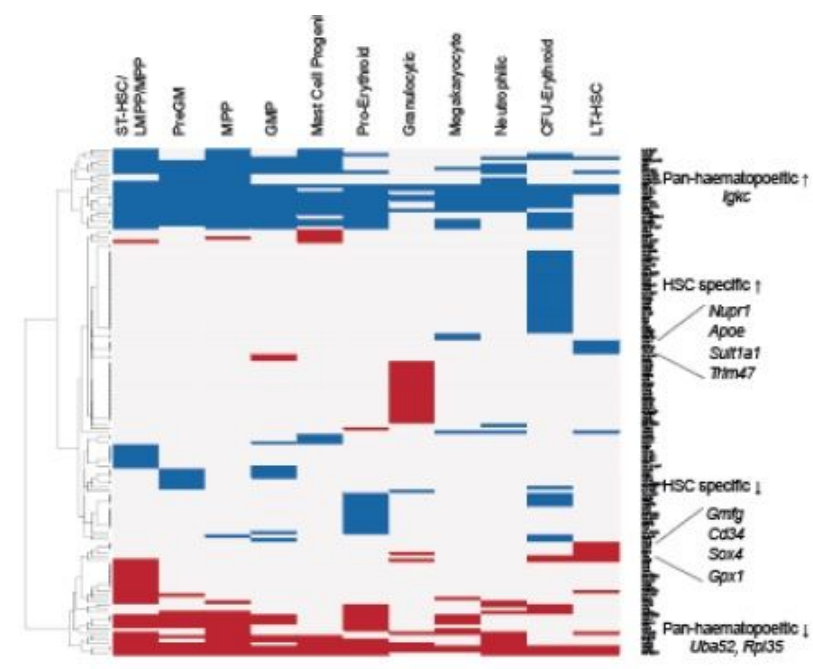

G

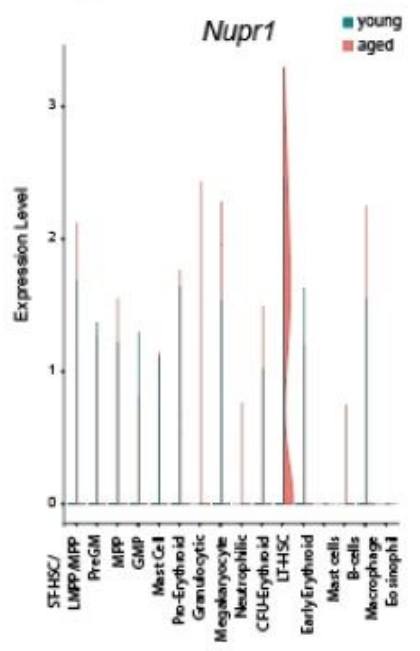

$\mathrm{H}$

$=8$ Weets old LSK CD34-CD48-CD $150+$ $\square 72$ Weeks oid LSK CD34-CD48-CD150+

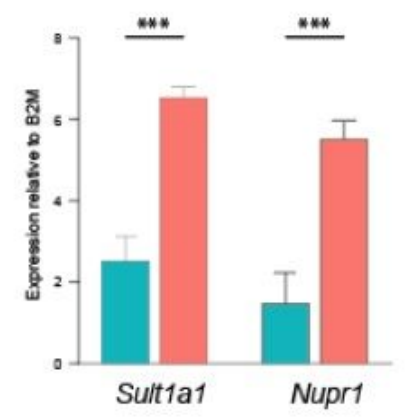

Figure 5

Cell frequency and gene expression changes in aged hematopoiesis A) Frequency of cells per cluster between young and aged mice. B-C) Procr expressing cells (HSCs) in young and aged mice, respectively. D) Differential expression for each cluster, based on short read data. Data are presented as a heatmap of fold-change values, where blue is upregulated with age and red is downregulated with age. E) Differential expression in the LT-HSC cluster. F) Expression of Sult1a1 in all clusters young and aged mice from shortread data G) Expression of Nupr1 in all clusters in young and aged mice from short-read data. H) qPCR confirmation of Sult1a1 and Nupr1 upregulation in aged phenotypic stem cells ( $* \star \star$ indicates p-value < 0.001) 
A

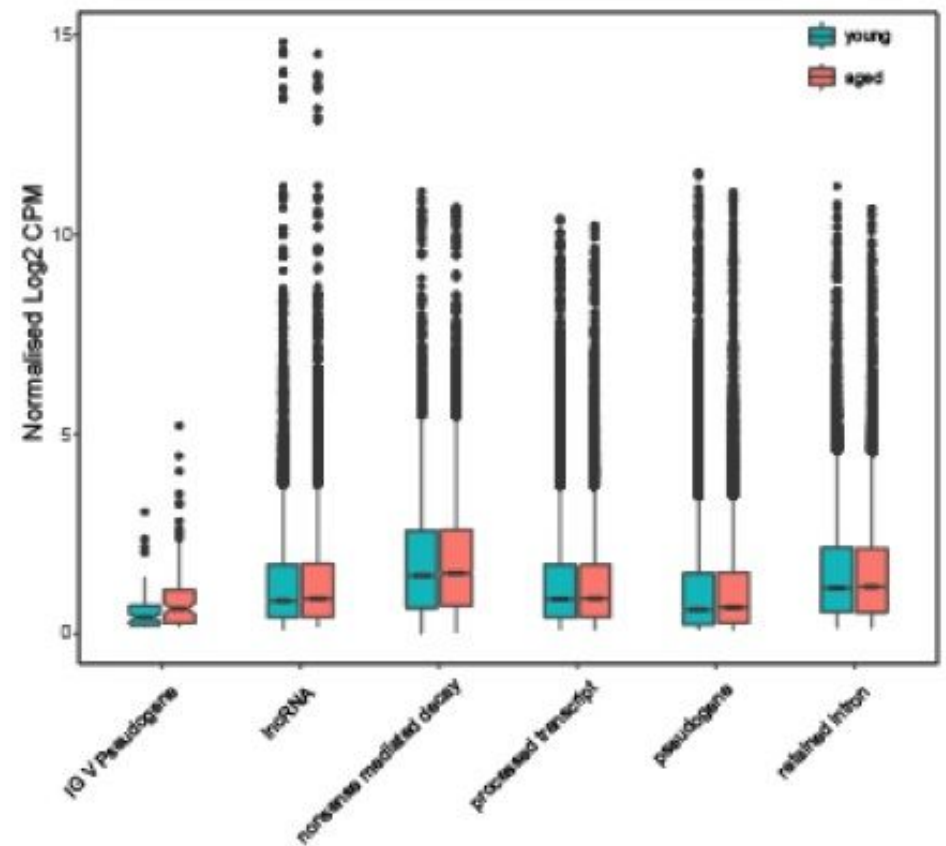

B

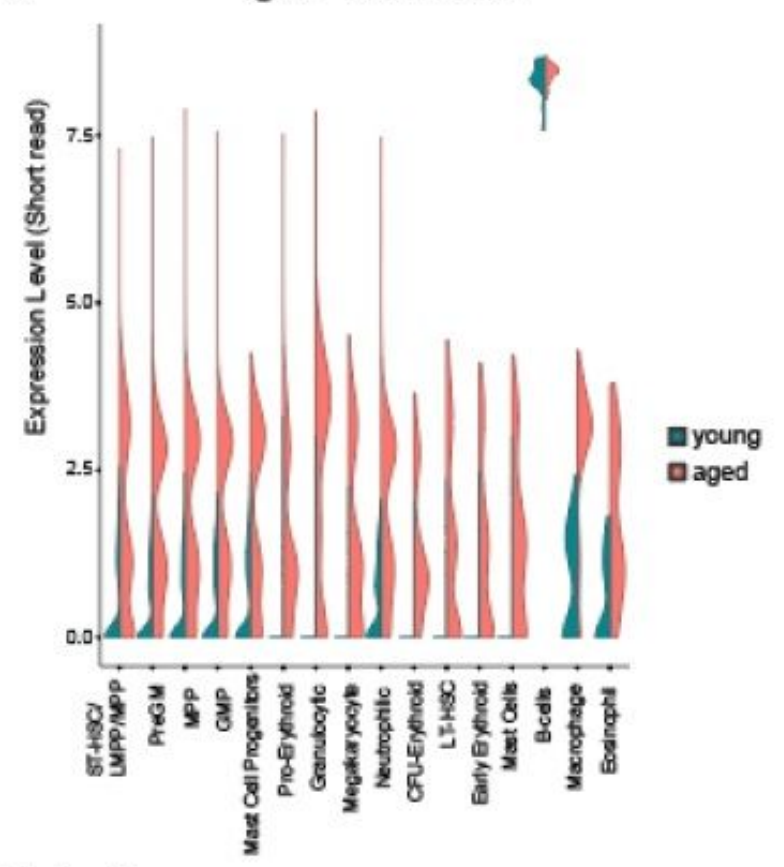

C

Igk - long read (HSC derived)

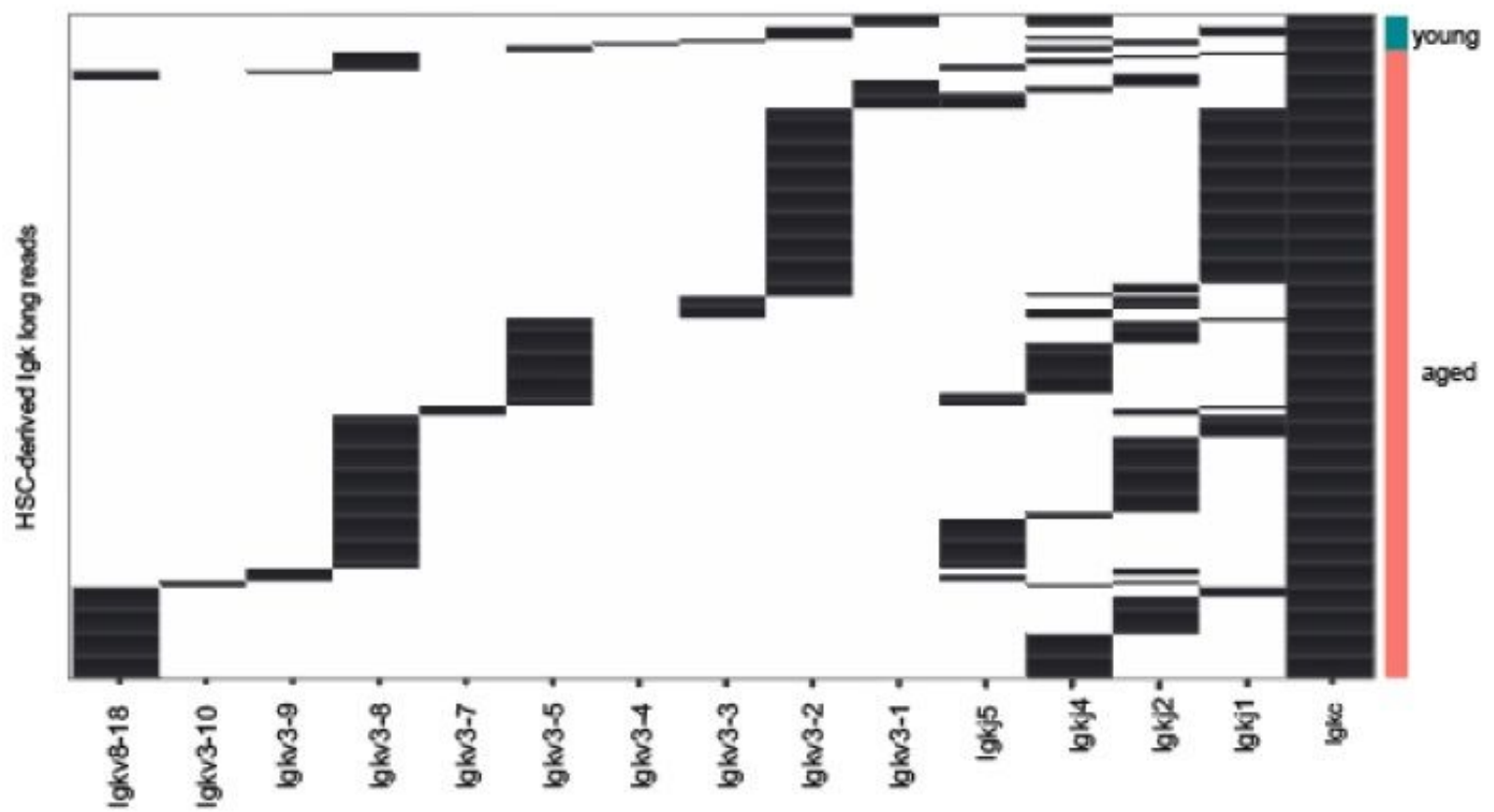

Figure 6

Integration of long- and short- read data to profile cell type isoform usage. Changes in classes of transcript between old and young mice, based on the long-read sequencing data B) Expression of Igkc from young and aged mice in all clusters from short-read data. C) Observation of full length, VJ recombined Igk transcripts in long-reads derived from the HSC cluster. Data are shown as a heatmap of long-reads (rows) with presence of $\mathrm{V}$ and $\mathrm{J}$ regions (columns) indicated in black.

\section{Supplementary Files}


This is a list of supplementary files associated with this preprint. Click to download.

- SupplementaryTable1.csv

- supplementaryfiguresandtables.pdf

- SupplementaryTable3.csv 